\title{
MAXIMAL ORDERS
}

\section{BY \\ MAURICE AUSLANDER AND OSCAR GOLDMAN(1)}

Introduction. This paper is about the structure theory of maximal orciers in two situations: over Dedekind rings in arbitrary simple algebras and over regular domains in full matrix algebras. By an order over an integrally closed noetherian domain $R$ we mean a subring $\Lambda$ of a central simple algebra $\Sigma$ over the quotient field $K$ of $R$ such that $\Lambda$ is a finitely generated $R$-module which spans $\Sigma$ over $K$. An order $\Lambda$ in the simple algebra $\Sigma$ is said to be maximal if $\Lambda$ if not properly contained in any order of $\Sigma$. The classical argument which shows that over a Dedekind ring every order is contained in a maximal one $[6$, p. 70$]$ is equally valid for arbitrary integrally closed noetherian domains.

After a preliminary study of maximal orders in general, we concentrate on maximal orders over discrete rank one valuation rings. The main results in this situation are as follows: all the maximal orders in a fixed simple algebra are conjugate, each is a principal ideal ring and is a full matrix algebra over a maximal order in a division algebra. Thus the theory of maximal orders over an arbitrary discrete rank one valuation ring is almost identical with the classical theory in which the valuation ring is assumed complete [6, Chapter VI]. A standard localization argument enables one to conclude that a maximal order over a Dedekind ring is the ring of endomorphisms of a finitely generated projective module over a maximal order in a division algebra.

A domain $R$ is said to be a regular domain if it is noetherian and the ring of quotients $R_{\mathfrak{p}}$ is a regular local ring for every prime ideal $\mathfrak{p}$ of $R$. The last section is devoted to proving the following: Let $R$ be a regular domain and $E$ a finitely generated $R$-module equal to its second dual with respect to $R$ : If the endomorphism ring of $E$ is $R$-projective then $E$ is $R$-projective. It follows from this that the $R$-projective maximal orders in a full matrix algebra over the quotient field of $R$ are precisely the endomorphism rings of finitely generated projective $R$-modules. As another consequence we obtain a new proof that a regular local ring is a unique factorization domain.

For ease of exposition we include in the Appendix some facts about modules and their endomorphism rings which are used in the course of the paper. Since many of these results are already in the literature (see for instance Curtis [5] and Morita [7]), we only sketch the proofs in most instances.

Many of the results contained in this paper will find application in our forthcoming publication on the theory of the Brauer group of commutative rings.

Received by the editors September 17, 1959.

(1) This work was carried out under a grant from the National Science Foundation. 
1. A characterization of maximal orders. Throughout this section, $R$ denotes a noetherian, integrally closed domain, $K$ denotes the field of quotients of $R$, and $\Sigma$ a fixed central simple $K$-algebra.

Let $M$ be a finitely generated $R$-submodule of $\Sigma$ such that $K M=\Sigma$. It is easily verified that the subset $\mathfrak{D}_{l}(M)$ of $\Sigma$ consisting of all $x$ in $\Sigma$ such that $x M \subset M$ is an order over $R$. The ring $\mathfrak{D}_{l}(M)$ is called the left order of $M$. Similarly, we define the right order of $M$ which we denote by $D_{r}(M)$. Since such a module $M$ can always be found in any simple algebra, it follows that every central simple algebra over $K$ contains at least one maximal order over $R$.

Proposition 1.1. If $\Omega$ is a subring of $\Sigma$ which is a finitely generated $R-$ module, then $\Omega$ is contained in a maximal order over $R$.

Proof. If $\Lambda$ is any order over $R$, then $\Lambda \Omega$ is a finitely generated $R$-module with the property that $K \Lambda \Omega=\Sigma$. Since $\Omega$ is a ring, we have that $\Omega$ is contained in $\mathfrak{D}_{r}(\Lambda \Omega)$. But we have already observed that $\mathfrak{D}_{r}(\Lambda \Omega)$ is contained in a maximal order over $R$, which completes the proof.

Let $\Lambda$ and $\Gamma$ be two orders over $R$ in $\Sigma$. By the conductor of $\Gamma$ with respect to $\Lambda$ we shall mean the set $\mathfrak{F}$ of all $x$ in $\Sigma$ such that $x \Gamma \subset \Lambda$. It is clear that $\mathfrak{F}$ is a left ideal in $\Lambda$ and is a right $\Gamma$-module. Furthermore it is clear that $\mathfrak{F} \cap R$ $\neq 0$.

Proposition 1.2. Let $\Lambda$ be a maximal order over $R$ and let $M$ be a multiplicative system in $R$ (i.e., $M \dot{M} \subset M$ and 0 not in $M$ ). Then $\Lambda \otimes_{R} R_{M}$ is a maximal order in $\Sigma$ over $R_{M}$.

Proof. It should first be remarked that $\Lambda \otimes_{R} R_{M}$ is an order over $R_{M}$ whenever $\Lambda$ is an order over $R$, whether $\Lambda$ is maximal or not.

Suppose $\Lambda$ is a maximal order and $\Gamma$ is an order over $R_{M}$ which contains $\Lambda \otimes R_{M}$. Since $\Gamma$ contains $\Lambda \otimes R_{M}$ it follows that the conductor $\mathfrak{F}$ of $\Gamma$ with respect to $\Lambda \otimes R_{M}$ is a right as well as a left ideal in $\Lambda \otimes R_{M}$. Thus $\mathfrak{F} \cap \Lambda$ is a right ideal in $\Lambda$. Since $\mathfrak{F} \cap R \neq 0$, we have that $K(\mathfrak{F} \cap \Lambda)=\Sigma$. Therefore $\mathfrak{D}_{r}(\mathfrak{F} \cap \Lambda)$ is an order over $R$ which contains $\Lambda$. Since $\Lambda$ is a maximal order we have the equality $\mathfrak{D}_{r}(\mathfrak{F} \cap \Lambda)=\Lambda$.

Now if $\gamma$ is in $\Gamma$, then $\mathfrak{F} \gamma$ is contained in $\Lambda \otimes R_{M}$. Thus we have that $(\mathfrak{F} \cap \Lambda) \gamma$ is rontained in $\Lambda \otimes R_{M}$. Since $(\mathfrak{F} \cap \Lambda) \gamma$ is a finitely generated $R$ module, it follows that there is an $m$ in $M$ such that (F $\cap \Lambda) \gamma m$ is contained in $\Lambda$. Also, it follows from the definition of $\mathfrak{F}$, that $(\mathfrak{F} \cap \Lambda) \gamma m$ is contained in $\mathfrak{F}$. Thus we have that $m \boldsymbol{\gamma}$ is in $\mathfrak{D}_{r}(\mathfrak{F} \cap \Lambda)$. Therefore, $m \gamma$ is in $\Lambda$, which means that $\gamma$ is in $\Lambda \otimes R_{M}$. This shows that $\Gamma=\Lambda \otimes R_{M}$ or $\Lambda \otimes R_{M}$ is a maximal order over $R_{M}$.

Corollary. An order $\Lambda$ over $R$ in $\Sigma$ is maximal if and only if $\Lambda \otimes R_{\mathfrak{m}}$ is maximal over $R_{\mathfrak{m}}$ for each maximal prime ideal $\mathfrak{m}$ in $R$. 
Proof. If $\Lambda$ is maximal, then by the preceding proposition we know that each $\Lambda \otimes R_{\mathfrak{m}}$ is a maximal order over $R_{\mathfrak{m}}$. On the other hand suppose $\Lambda \otimes R_{\mathfrak{m}}$ is maximal over $R_{\mathfrak{m}}$ for all maximal ideals in $R$ and that $\Gamma$ is an order containing $\Lambda$. Then $\Gamma \otimes R_{\mathfrak{m}}=\Lambda \otimes R_{\mathfrak{m}}$ or $\Gamma / \Lambda \otimes R_{\mathfrak{m}}=0$ for all maximal ideals $\mathfrak{m}$ in $R$. From this it follows that $\Gamma / \Lambda=0$ or $\Lambda$ is a maximal order over $R$.

If $\Lambda$ is an order over $R$ in the simple algebra $\Sigma$, we have that $K \Lambda=\Sigma$. It follows from this that every $R$-map from $\Lambda$ to $R$ has a unique extension to a $K$-map of $\Sigma$ to $K$. If we denote by $\Lambda^{*}$ the dual $\operatorname{Hom}_{R}(\Lambda, R)$ and by $\Sigma^{*}$ the dual $\operatorname{Hom}_{K}(\Sigma, K)$, then we have that $\Lambda^{*}$ is contained in $\Sigma^{*}$. It is also clear that $K \Lambda^{*}=\Sigma^{*}$, so that we may conclude that $\Lambda^{* *}$ is contained in $\Sigma^{* *}$. The canonical identification of $\Sigma^{* *}$ with $\Sigma$ therefore gives a canonical imbedding of $\Lambda^{* *}$ in $\Sigma$.

Proposition 1.3. If $\Lambda$ is an order over $R$, then $\Lambda^{* *}$ is also an order over $R$ and $\Lambda^{* *}$ contains $\Lambda$.

Proof. Making use of the identification of $\Sigma^{* *}$ with $\Sigma$, we may describe $\Lambda^{* *}$ as the set of all elements $x$ in $\Sigma$ with the property that $f(x)$ is in $R$ for all those $f$ in $\Sigma^{*}$ for which $f(\Lambda) \subset R$. It is clear from this that $\Lambda$ is contained in $\Lambda^{* *}$. It is also readily verified that $\Lambda^{* *}$ is a finitely generated $R$-module such that $K \Lambda^{* *}=\Sigma$. Thus to show that $\Lambda^{* *}$ is an order over $R$ it remains only to show that $\Lambda^{* *}$ is closed under multiplication. That is, we must show that if $f$ is in $\Sigma^{*}$ such that $f(\Lambda)$ is contained in $R$ and $x$ and $y$ are in $\Lambda^{* *}$, then $f(x y)$ is in $R$.

Now, $\Sigma^{*}$ is a two-sided $\Sigma$-module through the following operations: if $u$ is in $\Sigma$ and $g$ is in $\Sigma^{*}$, we set $(u g)(t)=g(t u)$ and $(g u)(t)=g(u t)$ for all $t$ in $\Sigma$. It is clear that if $f$ is in $\Lambda^{*}$ and $u$ is in $\Lambda$, then both $u f$ and $f u$ are also in $\Lambda^{*}$. Suppose $f$ is in $\Lambda^{*}$ and $x$ is in $\Lambda^{* *}$. If $t$ is in $\Lambda$, then $t f$ is in $\Lambda^{*}$ so that $(t f)(x)$ $=f(x t)$ is in $R$. But this shows that $f x$ is also in $\Lambda^{*}$. Thus if $y$ is in $\Lambda^{* *}$, then $(f x)(y)=f(x y)$ is in $R$. Therefore $\Lambda^{* *}$ is in fact closed under multiplication and is consequently an order over $R$.

Before proceeding to the main result of this section, which is a characterization of maximal orders, we state without proof (see, for instance, [3, Proposition 3.4]).

Proposition 1.4. Let $R$ be an integrally closed noetherian domain with field of quotients $K$. Suppose that $A \subset B$ are finitely generated torsion free $R$-modules such that $K \otimes A=K \otimes B$ and $A=A^{* *}$. If $A \neq B$, then $\operatorname{ann}(B / A)$, the annihilator of $B / A$, is an unmixed ideal of rank 1 .

THEOREM 1.5. An order $\Lambda$ over $R$ in $\Sigma$ is a maximal order if and only if $\Lambda=\Lambda^{* *}$ and $\Lambda \otimes_{R} R_{\mathfrak{p}}$ is a maximal order over $R_{\mathfrak{p}}$ for every minimal prime ideal $\mathfrak{p}$ of $R$.

Proof. In view of Propositions 1.2 and 1.3, the conditions are clearly necessary. 
Suppose now that $\Lambda=\Lambda^{* *}$ and $\Lambda \otimes R_{\mathfrak{p}}$ is maximal for all minimal prime ideals $\mathfrak{p}$ in $R$. If $\Gamma$ is an order containing $\Lambda$, then $\Gamma \otimes R_{\mathfrak{p}}=\Lambda \otimes R_{\mathfrak{p}}$ for all minimal prime ideals $\mathfrak{p}$ in $R$. Thus the ideal $\operatorname{ann}(\Gamma / \Lambda)$ is not of rank 1 . Since we are also assuming that $\Lambda=\Lambda^{* *}$ it follows from the previous proposition that $\Gamma=\Lambda$. Thus $\Lambda$ is a maximal order.

We have shown that the question of determining whether an order $\Lambda$ is maximal or not splits into two parts. The problem of showing that $\Lambda=\Lambda^{* *}$ is a purely module-theoretic question. The problem of determining whether or not $\Lambda \otimes_{R} R_{\mathfrak{p}}$ is maximal where $\mathfrak{p}$ is a minimal prime is the same as describing when an order over a discrete, rank one valuation ring is maximal, since the fact that $R$ is integrally closed guarantees that each $R_{\mathfrak{p}}$ is a discrete, rank one valuation ring. This problem is dealt with in the next two sections where we give a structure theory for maximal orders over discrete rank one valuation rings.

2. Maximal orders over Dedekind rings. Let $\mathfrak{O}$ be a discrete, rank one valuation ring, $\mathfrak{m}$ its maximal ideal and $K$ its field of quotients. Suppose $\Sigma$ is a central simple $K$-algebra and $\Lambda$ is an order in $\Sigma$. Since $m$ is the radical of $\mathfrak{D}$ and $\Lambda$ is a finitely generated $\mathfrak{D}$-module, it follows that $m \Lambda$ is contained in the radical $\mathfrak{N}$ of $\Lambda$. Also we have that $\Lambda / \mathrm{m} \Lambda$ is a finite dimensional $\mathfrak{D} / \mathrm{m}$ algebra. Thus there are only a finite number of maximal ideals in $\Lambda$ and $\mathfrak{N}^{i} \subset \mathrm{m} \Lambda$ for some $i$. Therefore $\bigcap_{i} \mathfrak{N}^{i}=0$ and the topologies induced by $\mathfrak{N}$ and $m \Lambda$ in $\Lambda$ are the same.

If $\Lambda$ is maximal we know that there exists a two sided $\Lambda$-submodule $\Re^{-1}$ of $\Sigma$ such that $\mathfrak{N} \mathfrak{N}^{-1}=\mathfrak{N}^{-1} \mathfrak{N}=\Lambda$ (see [6, p. 74]). In fact $\mathfrak{N}^{-1}$ consists precisely of all elements $x$ in $\Sigma$ such that $\mathfrak{N} \subset \subset \Lambda$. Since $\mathfrak{N}^{-1} \mathfrak{N}=\Lambda$ we have that there are $x_{i}$ in $\mathfrak{N}^{-1}$ and $y_{i}$ in $\mathfrak{R}$ such that $\sum x_{i} y_{i}=1$. Therefore if we define $f_{i}$ in $\operatorname{Hom}_{\Lambda}(\mathfrak{N}, \Lambda)$ by $f_{i}(z)=z x_{i}$, we have that $\sum f_{i}(z) y_{i}=z$ for all $z$ in $\mathfrak{N}$. Thus $\mathfrak{N}$ is a projective left $\Lambda$-module (it is similarly a projective right $\Lambda$-module). We also know that if $\Lambda$ is maximal, then $\mathfrak{N}$ is a maximal two-sided ideal in $\Lambda$ (see [6, p. 108]). Thus we have:

Proposition 2.1. If $\Lambda$ is an order over $\mathfrak{D}$ in a simple algebra and $\mathfrak{N}$ is the radical of $\Lambda$, then $\bigcap_{i} \mathfrak{N}^{i}=0$. If $\Lambda$ is a maximal order, then $\mathfrak{N}$ is a maximal twosided ideal and is projective as a left or right $\Lambda$-module.

We shall now derive some consequences of the fact that the radical $\mathfrak{N}$ of $\Lambda$ is left $\Lambda$-pinjective.

THEOREM 2.2. Let $\Lambda$ be an D-algebra which is a finitely generated torsionfree $\mathfrak{D}$-module such that the radical $\mathfrak{N}$ of $\Lambda$ is left $\Lambda$-projective. If $E$ is a finitely generated $\Lambda$-module, then hd $_{\Lambda} E=$ hdo $E$.

Proof. Since $D$ is a principal ideal ring and $\Lambda$ is a finitely generated torsion free $\mathfrak{D}$-module, it follows that $\Lambda$ is $\mathfrak{D}$-free. Thus we have that hd $\cos _{\Delta} E \geqq$ hdo $E$. In order to establish the reverse inequality it suffices to show that every 
finitely generated $\Lambda$-module which is $\mathfrak{D}$-free is $\Lambda$-projective. In order to do this we consider some special cases.

Suppose that $A$ is a finitely generated $\Lambda$-module such that $\mathfrak{R} A=0$. Then $A$ is a $\Lambda / \mathfrak{N}$-module and since $\Lambda / \mathfrak{N}$ is semi-simple we have that $A$ is a projective $\Lambda / \mathfrak{N}$-module. Now the hypothesis that $\mathfrak{N}$ is projective means that $\operatorname{hd}_{\Lambda} \Lambda / \mathfrak{N} \leqq 1$. From the general inequality $\operatorname{hd}_{\Lambda} A \leqq \operatorname{hd}_{\Lambda}(\Lambda / \mathfrak{R})+\operatorname{hd}_{\Lambda / \mathfrak{N}} A$, we have that $\operatorname{hd}_{\Lambda} A \leqq 1$.

Now suppose that $B$ is a finitely generated $\Lambda$-module such that $\mathfrak{m} B=0$. Then $B$ is a finite dimensional vector space over $D / \mathfrak{m}$ and thus has a composition series $B=B_{0} \supset B_{1} \supset \cdots \supset B_{n} \supset B_{n+1}=0$ over $\Lambda$. Since each $B_{i} / B_{i+1}(i=0, \cdots, n)$ is a simple $\Lambda$-module, we have that $\mathfrak{N}\left(B_{i} / B_{i+1}\right)=0$. Therefore $\operatorname{hd}_{\Lambda} B_{i} / B_{i+1} \leqq 1$ for $i=0, \cdots, n$. From this it follows that $\operatorname{hd}_{\Lambda} B$ $\leqq 1$.

Suppose that $C$ is a finitely generated $\Lambda$-module which is $\mathfrak{D}$-free. Because $C$ is $\mathfrak{D}$-free, it follows that $\pi$ is not a zero divisor for $C$ where $m=\pi \mathfrak{D}$. From the exact sequence $0 \rightarrow C \rightarrow{ }^{\pi} C \rightarrow C / \pi C \rightarrow 0$, we deduce the exact sequence

$$
\operatorname{Ext}_{\Lambda}^{1}(C / \pi C, D) \rightarrow \operatorname{Ext}_{\Lambda}^{1}(C, D) \stackrel{\pi}{\rightarrow} \operatorname{Ext}_{\Lambda}^{1}(C, D) \rightarrow \operatorname{Ext}_{\Lambda}^{2}(C / \pi C, D)
$$

for any $\Lambda$-module $D$. Since $\pi(C / \pi C)=0$, we have that $\operatorname{hd}_{\Lambda}(C / \pi C) \leqq 1$. Thus $\operatorname{Ext}_{\Lambda}^{2}(C / \pi C, D)=0$. Now if $D$ is a finitely generated $\Lambda$-module, then $\operatorname{Ext}_{\Lambda}^{1}(C, D)$ is a finitely generated $\mathfrak{D}$-module. Thus the fact that the map

$$
\operatorname{Ext}_{\Lambda}^{1}(C, D) \stackrel{\pi}{\rightarrow} \operatorname{Ext}_{\Lambda}^{1}(C, D)
$$

is an epimorphism means that $\operatorname{Ext}_{\Lambda}^{1}(C, D)=0$ for all finitely generated $\Lambda$ modules $D$. Since $\Lambda$ is noetherian and $C$ is finitely generated, it follows that $C$ is $\Lambda$-projective. The theorem now follows trivially.

COROLlaRY. If $\Lambda$ is an $\mathfrak{D}$-algebra which is a finitely generated torsion-free $\mathfrak{D}$-module such that $\mathfrak{N}$ is left $\Lambda$-projective, then $\Lambda$ is both left and right hereditary, i.e., every submodule of a projective module is projective.

Proof. Since $\Lambda$ is $\mathfrak{D}$-projective and $\mathfrak{D}$ is hereditary, it follows from Theorem 2.2 that each left ideal in $\Lambda$ is $\Lambda$-projective. Thus $\Lambda$ is left hereditary. Since $\Lambda$ is both left and right noetherian, it follows that $\Lambda$ is also right hereditary. (See [1, Theorem 4].)

THEOREM 2.3. Let $\Lambda$ be an order over $\mathfrak{D}$ in the simple algebra $\Sigma$. Then $\Lambda$ is a maximal order if and only if the radical $\mathfrak{N}$ of $\Lambda$ is a maximal two-sided ideal in $\Lambda$ and $\Lambda$ is hereditary.

Proof. If $\Lambda$ is maximal we have already observed that $\mathfrak{R}$ is a maximal two-sided ideal and $\mathfrak{N}$ is $\Lambda$-projective. Thus, from Theorem 2.2 it follows that $\Lambda$ is hereditary.

Suppose now that $\Lambda$ is hereditary and $\mathfrak{N}$, the radical of $\Lambda$, is a maximal 
two-sided ideal. Let $\Gamma$ be an order containing $\Lambda$. Then considering $\Gamma$ as a left $\Lambda$-module we have that $\operatorname{Hom}_{\Lambda}(\Gamma, \Gamma)$ can be identified with $\Gamma^{0}$, the opposite ring of $\Gamma$. Now the set of all $\sum f_{i}\left(\gamma_{i}\right)$ in $\Lambda$ where $f_{i}$ runs through $\operatorname{Hom}_{\Lambda}(\Gamma, \Lambda)$ and $\gamma_{i}$ runs through $\Gamma$ form a two-sided ideal in $\Lambda$ which is denoted by $\mathfrak{T}_{\Lambda}(\Gamma)$ (see Appendix). Since $\Gamma$ is $\mathfrak{D}$-projective, we have by Theorem 2.2 that $\Gamma$ is $\Lambda$-projective. Thus it follows that $\mathfrak{T}_{\Lambda}(\Gamma) \Gamma=\Gamma$. (See A.3.) This means that $\mathfrak{T}_{\Lambda}(\Gamma)=\Lambda$. For if $\mathfrak{T}_{\Lambda}(\Gamma) \neq \Lambda$, then $\mathfrak{T}_{\Lambda}(\Gamma)$ would be contained in $\Re$, the unique maximal two-sided ideal in $\Lambda$. Thus we would have that $\mathfrak{R} \Gamma=\Gamma$. But this can not happen since $\mathfrak{N}$ is the radical of $\Lambda$ and $\Gamma$ is a finitely generated, nonzero module. The fact that $\mathfrak{T}_{\Lambda}(\Gamma)=\Lambda$ implies that $\Lambda=\operatorname{Hom}_{\Gamma^{\circ}}(\Gamma, \Gamma)=\Gamma$ under the inclusion map (see A.2). Thus $\Lambda$ is a maximal order.

Before we can use this characterization of maximal orders over discrete, rank one valuation rings effectively we need the following fairly general lemma.

LEMMA 2.4. Let $R$ be an arbitrary commutative ring, $\Lambda$ an $R$-algebra which is left noetherian. Let $\Gamma$ be an R-algebra which is a flat $R$-module. Then if $A$ is a finitely generated left $\Lambda$-module and $B$ any left $\Lambda$-module we have that $\Gamma \otimes_{R} \operatorname{Ext}_{\Lambda}^{n}(A, B) \cong \operatorname{Ext}_{\Gamma \otimes_{R} \Lambda}^{n}\left(\Gamma \otimes_{R} A, \Gamma \otimes_{R} B\right)$ for all $n \geqq 0$.

Proof. If $C$ is any left $\Lambda$-module, define

$$
\alpha: \Gamma \otimes_{R} \operatorname{Hom}_{\Lambda}(C, B) \rightarrow \operatorname{Hom}_{\Gamma \otimes \Lambda}\left(\Gamma \otimes_{R} C, \Gamma \otimes_{R} B\right)
$$

by $\alpha(x \otimes f)(y \otimes c)=y x \otimes f(c)$ for all $x$ and $y$ in $\Gamma$, for all $f$ in $\operatorname{Hom}_{\Lambda}(C, B)$, and all $c$ in $C$. In case $C=\Lambda$ we have a commutative diagram

$$
\begin{array}{ccc}
\Gamma \otimes_{R} \operatorname{Hom}_{\Lambda}(\Lambda, B) & \stackrel{\alpha}{\rightarrow} \operatorname{Hom}_{\Gamma \otimes \Lambda}(\Gamma \otimes \Lambda, \Gamma \otimes B) \\
\downarrow & \downarrow \\
\Gamma \otimes_{R} B & = & \Gamma \otimes_{R} B
\end{array}
$$

where the vertical maps are the natural identifications. Thus $\alpha$ is an isomorphism when $C=\Lambda$ and therefore is also an isomorphism when $C$ is a finitely generated free $\Lambda$-module.

Since $A$ is a finitely generated left $\Lambda$-module and $\Lambda$ is left noetherian, we can find a free resolution $X$ of $A$ all of whose components are finitely generated free $\Lambda$-modules. Then

$$
\Gamma \otimes_{R} \operatorname{Hom}_{\Lambda}(X, B) \cong \operatorname{Hom}_{\Gamma \otimes \Lambda}(\Gamma \otimes X, \Gamma \otimes B)
$$

through $\alpha$. Passing to homology we obtain

$$
H\left(\Gamma \otimes_{R} \operatorname{Hom}_{\Lambda}(X, B)\right) \cong H\left(\operatorname{Hom}_{\Gamma \otimes \Lambda}(\Gamma \otimes X, \Gamma \otimes B)\right) .
$$

Since $\Gamma$ is $R$-flat we have that $\Gamma \otimes_{R} X$ is a free $\Gamma \otimes \Lambda$-resolution of $\Gamma \otimes A$ and also that $H\left(\Gamma \otimes_{R} \operatorname{Hom}_{A}(X, B)\right) \cong \Gamma \otimes_{R} H\left(\operatorname{Hom}_{A}(X, B)\right)$. Therefore 


$$
\Gamma \otimes \operatorname{Ext}_{\Lambda}^{n}(A, B) \cong \operatorname{Ext}_{\Gamma \otimes \Delta}^{n}(\Gamma \otimes A, \Gamma \otimes B)
$$

We will denote the completion of $\mathfrak{D}$ and $K$ (the field of quotients of $D$ ) by $\hat{D}$ and $\hat{K}$. If $\Sigma$ is a central simple $K$-algebra, then $\hat{\Sigma}=\hat{K} \otimes_{K} \Sigma$ is a central simple $\hat{K}$-algebra. Observing that $\hat{K}=\hat{\mathfrak{D}} \otimes \triangleright K$, we see that if $\Lambda$ is an $\mathcal{D}$-order in $\Sigma$, then $\hat{\Lambda}=\Lambda \otimes \circ \hat{\mathcal{D}}$ is an order in $\hat{\Sigma}$. Denoting the completion of $\mathfrak{m}$ by $\hat{m}$, we have that $\hat{\mathrm{m}} \hat{\Lambda}$ is contained in the radical of $\hat{\Lambda}$. Since $\hat{\Lambda} / \hat{\mathrm{m}} \hat{\Lambda} \cong \Lambda / \mathrm{m} \Lambda$ we see that $\Lambda$ has a unique maximal two-sided ideal if and only if $\hat{\Lambda}$ has only one maximal two-sided ideal.

Also it is clear that if $\mathfrak{N}$ denotes the radical of $\Lambda$, then $\hat{\mathfrak{R}}=\mathfrak{R} \otimes 0 \hat{\mathcal{S}}$ is the radical of $\hat{\Lambda}$. Thus if $\mathfrak{N}$ is $\Lambda$-projective, we see that $\hat{\mathfrak{N}}$ is $\hat{\Lambda}$-projective. Also we know that $\hat{\mathfrak{D}}$ is $\mathfrak{D}$-flat. (See [2, Proposition 3.1].) Therefore we have by the previous lemma that

$$
\hat{\mathfrak{D}} \otimes \operatorname{Dxt}_{\Lambda}^{n}(\mathfrak{N}, B) \cong \operatorname{Ext}_{\hat{\Lambda}}^{n}(\hat{\mathfrak{N}}, \hat{\mathfrak{D}} \otimes B)
$$

for all $n \geqq 0$. Thus $\hat{\mathfrak{D}} \otimes{ }_{0} \operatorname{Ext}_{\Lambda}^{n}(\mathfrak{R}, B)=0$, if $\hat{\mathfrak{N}}$ is $\hat{\Lambda}$-projective. In particular if $B$ is a finitely generated $\Lambda$-module, then $\operatorname{Ext}_{\Lambda}^{n}(\mathfrak{N}, B)$ is a finitely generated $\mathfrak{D}$-module which means that $\operatorname{Ext}_{\Lambda}^{n}(\mathfrak{N}, B)=0$ for all $n \geqq 0$ since $\operatorname{Ext}_{\Lambda}^{n}(\mathfrak{N}, B)$ is contained in its completion. Since $\Lambda$ is noetherian, this shows that $\mathfrak{N}$ is $\Lambda$ projective if and only if $\hat{\mathfrak{N}}$ is $\hat{\Lambda}$-projective. Combining these remarks with the characterization of maximal orders given in Theorem 2.3, we obtain the classical result, (see [6, Chapter VI]):

Proposition 2.5. An order $\Lambda$ is maximal over $\mathfrak{D}$ if and only if $\hat{\Lambda}$ is maximal over $\hat{\mathfrak{D}}$.

We end this section by giving a few applications of Theorem 2.2 to the theory of orders over arbitrary Dedekind rings.

Proposition 2.6. Let $R$ be an arbitrary commutative ring, $\Lambda$ an $R$-algebra which is a (left) noetherian ring. If $E$ is a finitely generated (left) $\Lambda$-module, then $\operatorname{hd}_{\Lambda} E=\sup _{\mathfrak{m}} \operatorname{hd}_{\Lambda \otimes R_{\mathfrak{m}}}\left(E \otimes R_{\mathfrak{m}}\right)$ where $\mathfrak{m}$ runs through all maximal ideals in $R$.

Proof. Since $R_{\mathfrak{m}}$ is $R$-flat we have that if $X$ is a $\Lambda$-projective resolution of $E$, then $X \otimes R_{\mathfrak{m}}$ is a $\Lambda \otimes R_{\mathfrak{m}}$-projective resolution of $E \otimes R_{\mathfrak{m}}$. Thus hd $\Lambda$ $\geqq \mathrm{hd}_{\Delta \otimes R_{\mathfrak{m}}} E \otimes R_{\mathfrak{m}}$ for all maximal ideals $\mathfrak{m}$ in $R$.

By Lemma 2.4 we have that

$$
R_{\mathfrak{m}} \otimes \operatorname{Ext}_{\Lambda}^{n}(E, B) \cong \operatorname{Ext}_{\Delta \otimes R_{\mathfrak{m}}}^{n}\left(E \otimes R_{\mathfrak{m}}, B \otimes R_{\mathfrak{m}}\right)
$$

for all $n \geqq 0$ and all $\Lambda$-modules $B$. Since an $R$-module $A$ is zero if and only if $R_{\mathfrak{m}} \otimes A=0$ for all maximal ideals $\mathfrak{m}$ in $R$, it follows from $\left(^{*}\right)$ that

$$
\sup _{\mathfrak{m}} \operatorname{hd}_{\Delta \otimes R_{\mathfrak{m}}}\left(E \otimes R_{\mathfrak{m}}\right) \geqq \mathrm{hd}_{\Lambda} E \text {. }
$$

Thus we obtain the desired equality. 
COROLlARY. Let $R$ be an arbitrary commutative ring, $\Lambda$ a left noetherian $R$-algebra. Then l.gl. $\operatorname{dim} \Lambda=\sup _{\mathfrak{m}}$ l.gl. $\operatorname{dim} \Lambda \otimes R_{\mathfrak{m}}$ where $\mathfrak{m}$ runs through all maximal ideals in $R$.

Proof. If follows immediately from the previous proposition that l.gl. $\operatorname{dim} \Lambda$ $\leqq$ sup $_{\mathfrak{m}}$ l.gl. $\operatorname{dim} \Lambda \otimes R_{\mathfrak{m}}$ where $\mathfrak{m}$ runs through all maximal ideals in $R$.

Now l.gl. $\operatorname{dim} \Lambda \otimes R_{\mathfrak{m}}=\operatorname{supl}\left(\Lambda \otimes R_{\mathfrak{m}}\right) / \mathfrak{l}$ (see [1, Theorem 1]) where $\mathfrak{l}$ runs through all left ideals in $\Lambda \otimes R_{\mathfrak{m}}$. But $\Lambda \otimes R_{\mathfrak{m}} / \mathfrak{l}=\Lambda / \Im \otimes R_{\mathfrak{m}}$ where $\Im=\mathfrak{l} \cap \Lambda$. Again by the previous proposition we know that hd $\Lambda / \Im \geqq h d_{\Lambda \otimes R_{\mathfrak{m}}} \Lambda / \Im \otimes R_{\mathfrak{m}}$. Thus l.gl. $\operatorname{dim} \Lambda \geqq \sup _{\mathfrak{m}}$ l.gl. $\operatorname{dim} \Lambda \otimes R_{\mathfrak{m}}$.

We now prove the global analogue of Theorem 2.2.

Proposition 2.7. Let $R$ be a Dedekind ring, $\Lambda$ an hereditary $R$-algebra which is a finitely generated, torsion free $R$-module. If $E$ is a finitely generated $\Lambda$ module, then hd $_{\Delta} E=$ hd $_{R} E$.

Proof. By Proposition 2.6 we know that $\operatorname{hd}_{\Delta} E=\sup h_{\mathfrak{m}_{\Delta \otimes R_{\mathfrak{m}}}}\left(E \otimes R_{\mathfrak{m}}\right)$. Since $\Lambda$ is hereditary (i.e., gl. $\operatorname{dim} \Lambda \leqq 1$ ) and noetherian, it follows from the above corollary that each $\Lambda \otimes R_{\mathrm{m}}$ is hereditary. Thus by Theorem 2.2 we have that $\operatorname{hd}_{\Delta \otimes R_{\mathfrak{m}}} E \otimes R_{\mathfrak{m}}=$ hd $_{R_{\mathfrak{m}}} E_{\mathfrak{m}}$. But hd $\mathrm{d}_{R} E=\operatorname{supm}_{\mathfrak{m}} \mathrm{hd}_{R_{\mathfrak{m}}} E \otimes R_{\mathfrak{m}}$ which gives that hd $_{\mathrm{A}} E=\operatorname{hd}_{R} E$.

Proposition 2.8. Let $R$ be a Dedekind ring with field of quotients $K$. Let $\Lambda$ be an hereditary $R$-algebra which is a finitely generated, torsion free $R$-module. $A$ finitely generated projective $\Lambda$-module $E$ is indecomposable if and only if $E \otimes_{R} K$ is a simple $\Lambda \otimes K-$ module.

Proof. If $E \otimes_{R} K$ is a simple $\Lambda \otimes K$-module, then it is clear that $E$ can not be split into a direct sum of $\Lambda$-modules in a nontrivial fashion, i.e., $E$ is indecomposable.

Suppose on the other hand that $E \otimes K$ is not $\Lambda \otimes K$-simple. Let $F_{0}$ be a proper nonzero submodule of $E \otimes K$. Then $E_{0}=F_{0} \cap E$ ( $E$ is contained in $E \otimes K$ since $E$ is projective and therefore torsion free over $R$ ) is a proper, nonzero $\Lambda$-submodule of $E$. But $E / E_{0}$ is $R$-torsion free since it is contained in the vector space $E \otimes K / F_{0}$. Thus $E / E_{0}$ is $R$-projective and therefore $\Lambda$ projective by Proposition 2.7. From this it follows that $E_{0}$ is a direct summand of $E$ or $E$ is not indecomposable.

TheORem 2.9. Let $R$ be a Dedekind ring with field of quotients $K$. If $\Lambda$ is an order over $R$ in the central simple $K$-algebra $\Sigma$, then $\Lambda$ is maximal if and only if $\Lambda$ is hereditary and given any nonzero prime ideal $\mathfrak{p}$ in $R$, there is one and only one maximal two-sided ideal $\mathfrak{P}$ in $\Lambda$ containing $\mathfrak{p}$.

Proof. It is easily seen that there will be only one maximal two-sided ideal lying over each nontrivial prime ideal in $R$ if and only if $\Lambda \otimes R_{\mathfrak{p}}$ has only one maximal two-sided ideal. We have already seen that $\Lambda$ is hereditary if and 
only if $\Lambda \otimes R_{\mathfrak{p}}$ is hereditary for each nontrivial prime ideal $\mathfrak{p}$ in $R$. Combining these observations with the characterization of maximal orders over discrete, rank one valuation rings given in Theorem 2.3 and the fact that $\Lambda$ is maximal if and only if $\Lambda \otimes R_{\mathfrak{p}}$ is maximal for all nontrivial prime ideals $\mathfrak{p}$ in $R$, we obtain the desired theorem.

REMARK. That not every hereditary order is maximal can be shown by a simple example. Let $k$ be the field of real numbers, $K=k(x)$. Also, set $p=x k[x]$ and $R=k[x]_{\mathrm{p}}$. Let $\Sigma$ be the crossed-product formed from the cyclic extension $K\left(x^{1 / 2}\right) / K$ and the unit factor set, so that $\Sigma$ is a full matrix algebra over $K$. Explicitly, $\Sigma$ is the algebra spanned over $K$ by $1, \alpha, \beta, \alpha \beta$ with the relations $\alpha^{2}=x, \beta^{2}=1, \alpha \beta=-\beta \alpha$.

Now let $\Lambda$ be the order in $\Sigma$ spanned over $R$ by $1, \alpha, \beta, \alpha \beta$. The radical of $\Lambda$ is $\Lambda \alpha$, as is easily verified from the fact that $\Lambda \alpha$ is a two-sided ideal and that the radical of $\Lambda / x \Lambda$ is the image of $\Lambda \alpha$. Furthermore, the left annihilator of $\alpha$ is 0 , so that the radical $\Lambda \alpha$ is a free $\Lambda$-module. The corollary to Theorem 2.2 then shows that $\Lambda$ is hereditary. However, $\Lambda \alpha$ is not a maximal two-sided ideal, for example, the left ideal generated by $\alpha$ and $\beta+1$ is two-sided and properly contains $\Lambda \alpha$. It follows from Proposition 2.1 that $\Lambda$ is not a maximal order.

3. Structure theorems. We have seen that a maximal order over a discrete rank one valuation ring has the property that modulo its radical it is a simple ring with minimum condition. A ring with this property is called a primary ring.

The following proposition is essentially known, but we include the proof for the sake of completeness.

Proposition 3.1. Let $\Omega$ be a finite dimensional primary algebra over a field $K$ and $E$ a finitely generated projective $\Omega$-module. Then $E$ can be generated as a module over $\Omega$ by $n$ elements if, and only if, $\operatorname{dim}_{K} E \leqq n \operatorname{dim}_{K} \Omega$. Furthermore, $E$ is free over $\Omega$ if, and only if, $\operatorname{dim}_{K} \Omega$ divides $\operatorname{dim}_{K} E$.

Proof. If $E$ can be generated by $n$ elements, then certainly $\operatorname{dim}_{K} E$ $\leqq n \operatorname{dim}_{K} \Omega$. Also, if $E$ is a free $\Omega$-module, then certainly $\operatorname{dim}_{K} \Omega$ divides $\operatorname{dim}_{K} E$.

Let $\mathfrak{R}$ be the radical of $\Omega$. Then, it is well-known that there are elements $e_{1}, e_{2}, \cdots, e_{r}$ in $\Omega$ such that (a) $e_{i}^{2}=e_{i}$, (b) $e_{i} e_{j}=0$, for $i \neq j$ (c) $e_{1}+\cdots+e_{r}$ $=1$, (d) $\bmod \mathfrak{N}$ the $e_{i}$ form a system of primitive idempotents in $\Omega / \mathfrak{N}$. The ideals $\Omega e_{i}$, being direct summands of $\Omega$, are projective $\Omega$-modules which are isomorphic mod $\mathfrak{R}$ (since $\Omega / \mathfrak{N}$ is a simple ring), so that they are isomorphic $\Omega$-modules. Now, $E / \mathfrak{N} E$ is a finitely generated module over the simple ring $\Omega / \mathfrak{R}$, so that $E / \mathfrak{R} E$ is isomorphic to a finite direct sum of modules of the form $\Omega / \mathfrak{N} \bar{e}_{i}$. (We denote the image in $\Omega / \mathfrak{N}$ of an element $x \in \Omega$ by $\bar{x}$.) Thus, we have a commutative diagram: 


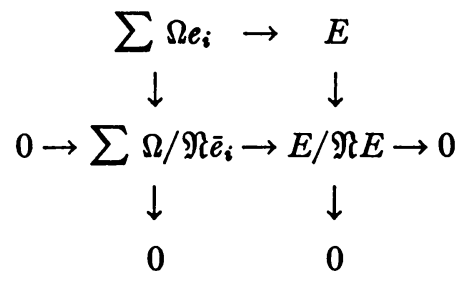

with exact rows and columns because $\sum \Omega e_{i}$ (direct sum) is projective. Since $\operatorname{Im}\left(\sum \Omega e_{i} \rightarrow E\right)+\Re E=E$, it follows that the top row is an epimorphism. By a similar argument, we obtain an epimorphism $E \rightarrow \sum \Omega e_{i}$, so that we conclude that $E \cong \sum \Omega e_{i}$.

Suppose that $E$ is the direct sum of $h$ copies of $\Omega e_{i}$. Remembering that $\Omega=\sum_{1}^{r} \Omega e_{i}$, write $h=q r+c$, with $0 \leqq c<r$. If $c=0$, then clearly $E$ is isomorphic to a direct sum of $q$ copies of $\Omega$ so that $E$ is a free $\Omega$-module with $q=\operatorname{dim}_{K} E / \operatorname{dim}_{K} \Omega$ generators. If $c \neq 0$, then $E$ can be generated by $q+1$ elements since a direct sum of $c \leqq r$ copies of $\Omega e_{i}$ is isomorphic to the ideal generated by $e_{1}+e_{2}+\cdots+e_{c}$. If $\operatorname{dim}_{K} E \leqq n \operatorname{dim}_{K} \Omega$, then $h \leqq n r$. Because $c \neq 0$, we have $h<n r$, so that $q \leqq n-1$, i.e., $E$ can be generated $\leqq n$ elements.

COROLlaRy. Let $R$ be a local ring with maximal ideal $\mathfrak{m}$, and $\Lambda$ a primary $R$-algebra which is a finitely generated $R$-module. Let $E$ be a finitely generated projective $\Lambda$-module. Then, $E$ can be generated over $\Lambda$ by $n$ elements if, and only if, $[E / \mathfrak{m} E: R / \mathfrak{m}] \leqq n[\Lambda / \mathfrak{m} \Lambda: R / \mathfrak{m}]$. $E$ is free on $n$ generators if, and only if, $[E / \mathfrak{m} E: R / \mathfrak{m}]=n[\Lambda / \mathfrak{m} \Lambda: R / \mathfrak{m}]$.

Proof. The result follows by combining the previous proposition with the observation that if $F$ is a submodule of a finitely generated $R$-module $E$ such that $E=F+\mathrm{m} E$, then $F=E$.

Lemma 3.2. Let $R$ be a noetherian (commutative) ring and $F_{1} \subset F_{2}$ finitely generated free $R$-modules. Then the rank of $F_{1}$ does not exceed the rank of $F_{2}$.

Proof. Let $\mathfrak{p}$ be a prime ideal belonging to zero in $R$, so that every nonunit in $R_{\mathfrak{p}}$ is a zero divisor. If $E=F_{2} / F_{1}$, then $\mathrm{hd}_{R} E \leqq 1$. We have the exact sequence $0 \rightarrow F_{1} \otimes R_{\mathfrak{p}} \rightarrow F_{2} \otimes R_{\mathfrak{p}} \rightarrow E \otimes R_{\mathfrak{p}} \rightarrow 0$ so that $\mathrm{hd}_{R_{\mathfrak{p}}}\left(E \otimes R_{\mathfrak{p}}\right) \leqq 1$. Because the nonunits in $R_{\mathfrak{p}}$ are zero divisors, it follows that $E \otimes R_{\mathfrak{p}}$ is $R_{\mathfrak{p}}$-free and therefore the sequence splits. We have therefore

$$
\operatorname{rank}_{R} F_{1}=\operatorname{rank}_{R_{\mathfrak{p}}}\left(F_{1} \otimes R_{\mathfrak{p}}\right) \leqq \operatorname{rank}_{R_{\mathfrak{p}}}\left(F_{2} \otimes R_{\mathfrak{p}}\right)=\operatorname{rank}_{R} F_{2} .
$$

By combining the lemma just proved with the corollary above, we see that we have also proved the following:

Proposition 3.3. Let $R$ be a local ring with maximal ideal $m$ and $\Lambda$ a primary $R$-algebra which is a finitely generated free $R$-module. If $E_{1} \subset E_{2}$ are finitely generated projective $\Lambda$-modules, then the number of elements required to 
generate $E_{1}$ is at most equal to the number of elements required to generate $E_{2}$. Consequently, every projective left (or right) ideal in $\Lambda$ is principal.

As an immediate consequence of the above proposition and the characterization of maximal orders over valuation rings given in the previous section, we have:

CoRollary. A maximal order over a discrete rank one valuation ring is a principal ideal ring.

In order to deduce a first consequence of this corollary, we need a lemma.

LEMMA 3.4. Let $R$ be a noetherian domain and $\Sigma$ a central simple algebra over the quotient field $K$ of $R$. Let $\Gamma$ be a maximal order in $\Sigma$ and $\Lambda$ an order such that conductor $\mathfrak{F}=\{x \in \Sigma \mid x \Gamma \subset \Lambda\}$ is a principal left ideal $\Lambda t$ in $\Lambda$. Then $t$ is a unit in $\Sigma$ and $\Lambda=t \Gamma t^{-1}$. Hence, in particular, $\Lambda$ is maximal.

Proof. Since both $\Lambda$ and $\Gamma$ are orders, we have $\mathfrak{F} \cap R \neq 0$, from which it follows that $t$ is a unit in $\Sigma$. Since $F$ is a right $\Gamma$-module, we have $t \Gamma \subset \Lambda t$ or $\Gamma \subset t^{-1} \Lambda t$. The maximality of $\Gamma$ gives the equality $\Gamma=t^{-1} \Lambda t$.

As an immediate consequence of this lemma, we have:

Proposition 3.5. Any two maximal orders over a discrete rank one valuation ring in the same simple algebra are isomorphic under an inner automorphism of tho simple algebra.

Let $\mathcal{O}$ be a discrete rank one valuation ring and $\Lambda$ a maximal order in a central simple algebra $\Sigma$ over the quotient field $K$ of $D$. If $M$ is a $\Lambda$-module which is finitely generated and torsion free over $\mathfrak{D}$, then $M$ is $\mathfrak{D}$-free, and

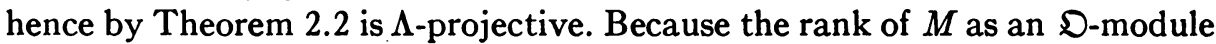
is finite, it follows that $M$ is a direct sum of a finite number of indecomposable $\Lambda$-submodules, each of which is again $\Lambda$-projective.

Let $E$ be an indecomposable $\Lambda$-module which is finitely generated and torsion free over $\mathfrak{D}$. Since $E$ is $\Lambda$-projective, we have by Proposition A.3 of the appendix, that $\mathfrak{T}_{\Lambda}(E) E=E$. Now $\mathfrak{T}_{\Lambda}(E)$ is a two-sided ideal in $\Lambda$, and the radical $\mathfrak{R}$ of $\Lambda$ is the unique maximal two-sided ideal of $\Lambda$. Since we cannot

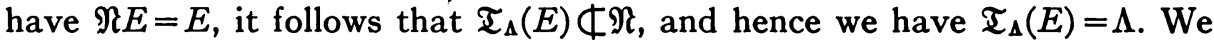
may now apply various conclusions of the appendix, principally A.2, A.3 and A.5 as follows. Set $\Omega=\operatorname{Hom}_{\Lambda}(E, E)$. Then $E$ is a finitely generated projective $\Omega$-module, and $\operatorname{Hom}_{\Omega}(E, E)=\Lambda$. By Proposition 2.8 we have that $K \otimes E$ is an indecomposable $\Sigma$-module, so that $\Omega$ is an order in $\Delta=\operatorname{Hom}_{\Sigma}(K \otimes E, K \otimes E)$, with $\Delta$ the division algebra equivalent to $\Sigma^{0}$, the opposite algebra of $\Sigma$. Now, by A.5, the two-sided ideals of $\Omega$ are in one-to-one correspondence with the two-sided ideals of $\Lambda$ in such a way that the radical of $\Omega$ is a maximal twosided ideal and is left $\Omega$-projective. Therefore, by Theorem 2.3 , it follows that $\Omega$ is a maximal order in $\Delta$. Summarizing, we have: 
THEOREM 3.6. If $\Lambda$ is a maximal order over a discrete rank one valuation ring $\mathfrak{D}$, and $E$ is an indecomposable $\Lambda$-module which is finitely generated and torsion-free over $\mathfrak{D}$, then $\Omega=\operatorname{Hom}_{\Delta}(E, E)$ is a maximal order in a division algebra and $\Lambda=\operatorname{Hom}_{\Omega}(E, E)$. Conversely, if $\Omega$ is a maximal order and $E$ is an $\Omega$-module which is finitely generated and torsion-free over $\mathfrak{D}$, then $\operatorname{Hom}_{\Omega}(E, E)$ is a maximal order.

We can carry the results of Theorem 3.6 still further by making use of the following:

Proposition 3.7. Let $\Lambda$ be a maximal order over $\mathfrak{D}$ in a simple algebra $\Sigma$. Then every indecomposable $\Lambda$-module which is finitely generated and torsion-free over $\mathfrak{D}$ is a cyclic $\Lambda$-module. If $\Sigma$ is a division algebra, then every finitely generated projective $\Lambda$-module is free over $\Lambda$.

Proof. Because $E$ is indecomposable and $\Lambda$-projective, we have, by Proposition 2.8, that $K \otimes E$ is a simple $\Sigma$-module and is therefore isomorphic to an ideal in $\Sigma$. Hence $E$ is isomorphic to an ideal in $\Lambda$, so that $E$ is cyclic because $\Lambda$ is a principal ideal ring. Now, if $\Sigma$ is a division algebra, a nonzero principal ideal of $\Lambda$ is a free $\Lambda$-module. It follows in this case that every finitely generated projective $\Lambda$-module is free.

By combining the two previous propositions, we have also proved the following structure theorem.

THEOREM 3.8. The maximal orders over a discrete rank one valuation ring are the full matrix algebras over maximal orders in division algebras.

By a suitable localization argument, the structure theorem may be extended to maximal orders over Dedekind rings.

THEOREM 3.9. If $\Lambda$ is a maximal order over a Dedekind ring $R$ in a simple algebra $\Sigma$, then there is a maximal order $\Omega$ in the division algebra $\Delta$ equivalent to $\Sigma^{0}$ as well as a finitely generated projective $\Omega$-module $E$ such that $\Lambda=\operatorname{Hom}_{\Omega}(E, E)$. Furthermore, $E$ is indecomposable and projective over $\Lambda$ and $\Omega=\operatorname{Hom}_{\Lambda}(E, E)$.

Proof. Let $M$ be a finitely generated projective $\Lambda$-module which is torsionfree over $R$, e.g., $M=\Lambda$. Then $M$ is a direct sum of indecomposable $\Lambda$ modules, each of which is again $\Lambda$-projective. Let $E$ be such a module, and let $\Omega=\operatorname{Hom}_{\Lambda}(E, E)$. By Proposition $2.8, K \otimes E$ is an indecomposable $\Sigma$ module, so that $\Omega$ is an order in $\Delta$.

If $\mathfrak{p}$ is any prime ideal of $R$ then $E \otimes R_{\mathfrak{p}}$ is a finitely generated projective indecomposable $\Lambda \otimes R_{\mathfrak{p}}$-module and $\Omega \otimes R_{\mathfrak{p}}$ is its endomorphism ring. Since $\Lambda \otimes R_{\mathfrak{p}}$ is a maximal order over $R_{\mathfrak{v}}$ (corollary to Proposition 1.2), it follows by Theorem 3.6 that $\Omega \otimes R_{\mathfrak{p}}$ is also a maximal order, and hence by the corollary to Proposition 1.2, that $\Omega$ is a maximal order over $R$.

Now $\mathfrak{T}_{\Lambda}(E)$ is a two-sided ideal in $\Lambda$. If $\mathfrak{T}_{\Lambda}(E)$ were a proper ideal, then 
it would be contained in a maximal two-sided ideal, and consequently, for a suitable prime ideal $\mathfrak{p}$ of $R$, we would have $\mathfrak{T}_{\Lambda}(E) \otimes R_{\mathfrak{p}}$ is a proper ideal in $\Lambda \otimes R_{\mathfrak{p}}$. But $\mathfrak{T}_{\Lambda}(E) \otimes R_{\mathfrak{p}}=\mathfrak{T}_{\Lambda \otimes R_{\mathfrak{p}}}\left(E \otimes R_{\mathfrak{p}}\right)$, and we know that $\mathfrak{T}_{\Lambda \otimes R_{\mathfrak{p}}}\left(E \otimes R_{\mathfrak{p}}\right)$ $=\Lambda \otimes R_{\mathfrak{p}}$. Thus, it follows that $\mathfrak{I}_{\Lambda}(E)=\Lambda$ and hence by $\mathrm{A} .2$ also that $\Lambda=\operatorname{Hom}_{\Omega}(E, E)$ and that $E$ is a finitely generated projective $\Omega$-module.

Returning again to the local situation, we have as a final result on modules over maximal orders, the following proposition.

Proposition 3.10. Let $\Lambda$ be a maximal order over a discrete rank one valua-

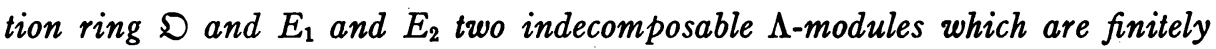
generated torsion-free over $\mathfrak{D}$. Then $E_{1}$ and $E_{2}$ are $\Lambda$-isomorphic. Consequently, the representation of $\Lambda$ in the form $\operatorname{Hom}_{\Omega}(E, E)$ with $\Omega$ a maximal order in a division algebra and $E$ a free $\Omega$-module uniquely determines $\Omega$ and $E$.

Proof. Set $\Omega=\operatorname{Hom}_{\Lambda}\left(E_{1}, E_{1}\right)$, so that $\Omega$ is a maximal order in a division algebra. Because $E_{1}$ is a finitely generated projective $\Lambda$-module, and $\mathfrak{T}_{\Lambda}\left(E_{1}\right)$ $=\Lambda$, it follows by Proposition A.4 of the appendix, that $E_{2}=M \otimes_{\Omega} E_{1}$, with $M$ a right $\Omega$-module. Since $E_{2}$ is $\Lambda$-projective, we have, again by proposition A.4, that $M$ is $\Omega$-projective, so that by Proposition 3.7, $M$ is $\Omega$-free. Since $E_{2}$ is $\Lambda$-indecomposable, the rank of $M$ over $\Omega$ must be one, which shows that $E_{2}$ is $\Lambda$-isomorphic to $E_{1}$. The second part of the statement of the proposition follows immediately from the first part.

We conclude the discussion of valuation rings with the following.

THEOREM 3.11. Let $\mathfrak{D}$ be a discrete rank one valuation ring and $\Lambda$ a maximal order in a central simple algebra over the quotient field $K$ of $\mathfrak{D}$. Let $\mathfrak{N}$ be the radical of $\Lambda$. Then the following statements are equivalent:

(a) $\Lambda / \mathfrak{N}$ is a division ring.

(b) All ideals in $\Lambda$ are two-sided and are powers of $\mathfrak{\Re}$.

(c) $K \otimes \Lambda$ is a division algebra and $\Lambda$ is the only maximal order in $K \otimes \Lambda$.

Proof. (a) $\Rightarrow(b)$. Let $x \neq 0$ be in $\Lambda$. Consider the exact sequence $0 \rightarrow \mathfrak{A}$ $\rightarrow \Lambda \rightarrow \Lambda x \rightarrow 0$ where $f(y)=y x$ and $\mathfrak{A}$ is the left annihilator of $x$. Since $\Lambda$ is maximal, every ideal is projective, so that the sequence splits and therefore $\mathfrak{A}=\Lambda e$ with $e$ an idempotent different from 1 . Because $\Lambda / \mathfrak{N}$ is a division algebra, we must have $e \in \mathfrak{R}$, and because $\bigcap_{\mathfrak{N}^{k}}=0$, we must have $e=0$. Thus, $\Lambda$ is an integral domain. Now $\mathfrak{N}$ is a principal left ideal $\mathfrak{N}=\Lambda \boldsymbol{u}$ as well as a principal right ideal. Because $\Lambda$ has no zero divisors, we have as a result that $\mathfrak{N}=u \Lambda$, and consequently also that $\mathfrak{N}^{k}=\Lambda u^{k}=u^{k} \Lambda$.

Let $\mathfrak{l}$ be a left ideal in $\Lambda$. If $\mathfrak{I} \subset \mathfrak{R}$, we have, because $\Lambda / \mathfrak{N}$ is a division algebra, that $\mathfrak{l}+\mathfrak{N}=\Lambda$ and therefore that $\mathfrak{l}=\Lambda$. Suppose now that $\mathfrak{I} \subset \mathfrak{N}$ and that $\mathfrak{l} \neq 0$. Since $\cap \mathfrak{N}^{k}=0$, there exists a smallest $k \geqq 2$ such that $\mathfrak{I} \subset \mathfrak{N}^{k}$. Now, $\mathfrak{N}^{k-1} / \mathfrak{N}^{k}=\Lambda u^{k-1} / \Lambda u^{k} \cong \Lambda / \Lambda u=\Lambda / \mathfrak{N}$, so that $\mathfrak{N}^{k-1} / \mathfrak{N}^{k}$ is a simple $\Lambda$-module. Now, we have $\mathfrak{I} \subset \mathfrak{N}^{k-1}$ and $\mathfrak{I} \subset \mathfrak{N}^{k}$ so that $\mathfrak{N}^{k} \subset \mathfrak{l}+\mathfrak{N}^{k} \subset \mathfrak{N}^{k-1}$ while $\mathfrak{l}+\mathfrak{N}^{k} \neq \mathfrak{N}^{k}$. It follows therefore that $\mathfrak{I}+\mathfrak{N}^{k}=\mathfrak{N}^{k-1}$ and therefore $\mathfrak{l}=\mathfrak{N}^{k-1}$. 
(b) $\Rightarrow$ (c). If $\mathfrak{p}$ is the maximal ideal of $\mathfrak{D}$, then $\mathfrak{N} \supset \mathfrak{p}$ so that $\mathfrak{N}^{k} \supset \mathfrak{p}^{k}$ and consequently $K \otimes \Lambda$ has no nontrivial left ideals. Thus $K \otimes \Lambda$ is a division algebra. Let $\Gamma$ be any maximal order in $K \otimes \Lambda$, and $\mathfrak{F}=\{x \in K \otimes \Lambda \mid x \Gamma \subset \Lambda\}$ the conductor of $\Gamma$ with respect to $\Lambda$. Since both $\Lambda$ and $\Gamma$ are maximal, we have $\mathfrak{D}_{l}(\mathfrak{F})=\Lambda$ and $\mathfrak{D}_{r}(\mathfrak{F})=\Gamma$. But $\mathfrak{F}$ is a left ideal in $\Lambda$ and hence also a right ideal in $\Lambda$. But this means that $\Lambda \subset \mathfrak{D}_{r}(\mathfrak{F})$, so that because of the maximality of $\Lambda$ we have $\Lambda=\Gamma$.

(c) $\Rightarrow(a)$. Let $\mathfrak{l}$ be a proper nonzero left ideal in $\Lambda$. Then $\mathfrak{D}_{r}(\mathfrak{l})$ is contained in some maximal order, and since $\Lambda$ is the only maximal order, $D_{r}(\mathfrak{l})$ must be contained in $\Lambda$. In other words, $\mathfrak{l}$ is a two-sided ideal, so that $\mathfrak{l} \subset \mathfrak{R}$. It follows that $\Lambda / \mathfrak{R}$ is a division ring.

CoROLlARY. Let $\mathfrak{D}$ be a complete discrete rank one valuation ring and $\Delta a$ central division algebra over the quotient field $K$ of $\mathfrak{D}$. If $\Lambda$ is a maximal order in $\Delta$, and $\mathfrak{N}$ is the radical of $\Lambda$, then $\Lambda / \mathfrak{N}$ is a division algebra, and consequently $\Lambda$ is the only maximal order in $\Delta$.

Proof. Because $\mathscr{D}$ is complete, every idempotent in $\Lambda / \mathfrak{N}$ can be lifted to $\Lambda$. Since $\Lambda / \mathfrak{N}$ is simple, and $\Delta$ is a division algebra, this shows that $\Lambda / \mathfrak{N}$ is a division algebra. It follows then by Theorem 3.11 that $\Lambda$ is the only maximal order in $\Delta$. (See [6, Theorem 12, p. 100].)

4. Maximal orders in a full matrix algebra. We begin this section with a proposition which will be used several times in the rest of the paper.

Proposition 4.1. Let $R$ be an integrally closed noetherian domain, and $A$ and $B$ finitely generated $R$-modules with $A$ torsion-free. Then the following isomorphisms hold:

$$
\operatorname{Hom}(A, B)^{* *} \cong\left(B^{*} \otimes A\right)^{*} \cong \operatorname{Hom}\left(A, B^{* *}\right) \cong \operatorname{Hom}\left(B^{*}, A^{*}\right) .
$$

In particular, it follows that

$$
\operatorname{Hom}(A, A)^{* *} \cong \operatorname{Hom}\left(A^{*}, A^{*}\right) \cong \operatorname{Hom}\left(A^{* *}, A^{* *}\right) \text {. }
$$

Proof. We define the map $\sigma: B^{*} \otimes A \rightarrow \operatorname{Hom}(A, B) *$ by $\sigma(f \otimes a)(g)=f(g(a))$, for $f \in B^{*}, a \in A$ and $g \in \operatorname{Hom}(A, B)$. The map $\sigma$ has a transpose, $\sigma^{*}: \operatorname{Hom}(A, B)^{* *} \rightarrow\left(B^{*} \otimes A\right)^{*}$. Let $\mathfrak{p}$ be a minimal prime ideal in $R$. From the hypotheses on $R$, it follows that $R_{\mathrm{p}}$ is a discrete rank one valuation ring and therefore that $A \otimes R_{\mathfrak{p}}$ is a finitely generated free $R_{\mathfrak{p}}$-module. From the fact that $R_{\mathrm{p}}$ is $R$-flat and the fact that $\sigma$ is an isomorphism whenever $A$ is $R$-free (see [4, Chapter VI, Proposition 5.2]), it follows from Lemma 2.4 that $\sigma \otimes 1: B^{*} \otimes A \otimes R_{\mathfrak{p}} \rightarrow \operatorname{Hom}(A, B)^{*} \otimes R_{\mathfrak{p}}$ is an isomorphism. Therefore the map $\sigma^{*} \otimes 1: \operatorname{Hom}(A, B)^{* *} \otimes R_{\mathrm{p}} \rightarrow\left(B^{*} \otimes A\right)^{*} \otimes R_{\mathrm{p}}$ is also an isomorphism. Since both $\operatorname{Hom}(A, B)^{* *}$ and $\left(B^{*} \otimes A\right)^{*}$ are torsion-free $R$-modules, we see that $\sigma^{*}$ is a monomorphism and that $K \otimes \operatorname{Hom}(A, B)^{* *} \cong K \otimes\left(B^{*} \otimes A\right)^{*}$, with $K$ the quotient field of $R$. Also $\operatorname{Hom}(A, B)^{* *}$ is equal to its own second dual, so that we may apply Proposition 1.4. Because $\sigma^{*} \otimes 1: \operatorname{Hom}(A, B)^{* *} \otimes R_{\mathfrak{p}} \rightarrow\left(B^{*} \otimes A\right)^{*} \otimes R_{\mathfrak{p}}$ 
is an isomorphism, it follows from Proposition 1.4 that $\sigma^{*}$ is an isomorphism. The rest of the isomorphisms are given by standard identities.

The fact that $\operatorname{Hom}(A, A)^{* *} \cong \operatorname{Hom}\left(A^{*}, A^{*}\right)$ follows from the first part of the proposition by setting $B=A$. Now, $\operatorname{Hom}(A, A)^{* *} \cong\left(\operatorname{Hom}(A, A)^{* *}\right)^{* *}$ $\cong\left(\operatorname{Hom}\left(A^{*}, A^{*}\right)\right)^{* *} \cong \operatorname{Hom}\left(A^{* *}, A^{* *}\right)$, which completes the proof.

The following proposition gives a description of the maximal orders in a full matrix algebra.

Proposition 4.2. Let $R$ be an integrally closed noetherian domain with quotient field $K$. Let $V$ be a finite dimensional vector space over $K$ and $\Sigma=\operatorname{Hom}_{K}(V, V)$. If $\Lambda$ is an order over $R$ in $\Sigma$, then $\Lambda$ is maximal if, and only if, there is a finitely generated $R$-submodule $E$ of $V$ such that $E=E^{* *}$ and $\Lambda=\operatorname{Hom}_{R}(E, E)$.

Proof. Suppose $\Lambda=\operatorname{Hom}_{R}(E, E)$ with $E=E^{* *}$. Then by the previous proposition, we have that $\Lambda^{* *}=\operatorname{Hom}\left(E^{* *}, E^{* *}\right)=\operatorname{Hom}(E, E)=\Lambda$. Also, if $\mathfrak{p}$ is a minimal prime ideal in $R$, then $\Lambda \otimes R_{\mathfrak{p}}=\operatorname{Hom}_{R_{\mathfrak{p}}}\left(E \otimes R_{\mathfrak{p}}, E \otimes R_{\mathfrak{p}}\right)$ with $E \otimes R_{\mathfrak{p}}$ a finitely generated free $R_{\mathfrak{p}}$-module. By Theorem 3.6 it follows that $\Lambda \otimes R_{\mathfrak{p}}$ is a maximal order over $R_{\mathfrak{p}}$, and hence by Theorem 1.5 , that $\Lambda$ is a maximal order.

Suppose now that $\Lambda$ is a maximal order. Let $v$ be a nonzero element of $V$, and define the $\Sigma$-epimorphism $\psi: \Sigma \rightarrow V$ by $\psi(\sigma)=\sigma(v)$, for all $\sigma \in \Sigma$. Then $E_{1}=\psi(\Lambda)$ is a finitely generated $R$-submodule of $V$, and $\operatorname{Hom}\left(E_{1}, E_{1}\right)$ is an order in $\Sigma$ which contains $\Lambda$. Using the usual identification of $V$ with $V^{* *}$, we have that $\dot{E}=E_{1}^{* *}$ is contained in $V$, contains $E_{1}$ and is finitely generated over $R$. Also, $\operatorname{Hom}(E, E)$ contains $\operatorname{Hom}\left(E_{1}, E_{1}\right)$. Since $\operatorname{Hom}(E, E)$ is an order containing $\Lambda$, it coincides with $\Lambda$. At the same time, it is clear that $E=E^{* *}$, which completes the proof of the proposition.

It is a classical result that the maximal orders in a full matrix algebra over the quotient field of a Dedekind ring are the endomorphism rings of finitely generated projective modules over the Dedekind ring. The rest of this paper is devoted to proving the following generalization of this classical result.

THEOREM 4.3. Let $R$ be a regular domain $\left({ }^{2}\right), K$ its field of quotients, $V a$ finite dimensional vector space over $K$ and $\Sigma=\operatorname{Hom}_{K}(V, V)$. Let $\Lambda$ be an order over $R$ in $\Sigma$ which is $R$-projective. Then $\Lambda$ is maximal if, and only if, $\Lambda=\operatorname{Hom}(E, E)$ with $E$ a finitely generated projective $R$-submodule of $V$.

In view of Proposition 4.2, in order to prove Theorem 4.3 it suffices to show that if a finitely generated module $E$ over a regular domain $R$ has the properties that $E=E^{* *}$ and $\operatorname{Hom}(E, E)$ is $R$-projective, then $E$ is $R$-projective. Consequently, by the usual localization procedure, proving the following Theorem 4.4 will also prove Theorem 4.3.

(2) See the introduction for the definition of regular domain. 
THEOREM 4.4. If $R$ is a regular local ring and $E$ is a finitely generated $R$ module such that $\operatorname{Hom}_{R}(E, E)$ is an $R$-free module, then the following statements are equivalent:

(a) $E$ is free.

(b) $E=E^{* *}$.

(c) $\operatorname{Ext}_{R}^{1}(E, E)=0$.

Proof. It is clear that (a) implies (b) and (c). In order to show that (c) implies (b), we nẹed a lemma.

LEMMA 4.5. Let $R$ be a noetherian ring and $E$ a finitely generated nonzero $R$-module. Then the prime ideals belonging to 0 in $E$ are the same as the prime ideals which belong to 0 in $\operatorname{Hom}(E, E)$. In particular, if $R$ is a noetherian domain, then $\operatorname{Hom}(E, E)$ is torsion-free if, and only if, $E$ is torsion-free.

Proof. Let $0 \rightarrow H \rightarrow F \rightarrow E \rightarrow 0$ be exact with $F$ a finitely generated free $R$-module. Then $0 \rightarrow \operatorname{Hom}(E, E) \rightarrow \operatorname{Hom}(F, E)$ is exact. Thus every prime ideal belonging to 0 in $\operatorname{Hom}(E, E)$ belongs to 0 in $\operatorname{Hom}(F, E)$. Since $\operatorname{Hom}(F, E)$ is a direct sum of copies of $E$, it follows that a prime ideal which belongs to 0 in $\operatorname{Hom}(E, E)$ also belongs to 0 in $E$.

Suppose $\mathfrak{p}$ belongs to 0 in $E$. Then there is an element $e \in E$ whose annihilator is exactly $\mathfrak{p}$, so that $e$ is not in the kernel of $E \rightarrow E \otimes R_{\mathfrak{p}}$, and therefore $E \otimes R_{\mathfrak{p}} \neq 0$. Since $\operatorname{Hom}_{R_{\mathfrak{p}}}\left(E \otimes R_{\mathfrak{p}} / \mathfrak{p} R_{\mathfrak{p}}, R_{\mathfrak{p}} / \mathfrak{p} R_{\mathfrak{p}}\right)$ is a submodule of $\operatorname{Hom}_{R_{\mathfrak{p}}}\left(E \otimes R_{\mathfrak{p}}, R_{\mathfrak{p}} / \mathfrak{p} R_{\mathfrak{p}}\right)$, it follows that $\operatorname{Hom}_{R_{\mathfrak{p}}}\left(E \otimes R_{\mathfrak{p}}, R_{\mathfrak{p}} / \mathfrak{p} R_{\mathfrak{p}}\right) \neq 0$ and therefore that $\operatorname{Hom}_{R}(E, R / \mathfrak{p}) \neq 0$. But the monomorphism $R / \mathfrak{p} \rightarrow E$ defined through the map $r \rightarrow r e$ induces a monomorphism $\operatorname{Hom}(E, R / \mathfrak{p}) \rightarrow \operatorname{Hom}(E, E)$. Thus $\mathfrak{p}$ belongs to 0 in $\operatorname{Hom}(E, E)$, since the annihilator of $\operatorname{Hom}(E, R / \mathfrak{p})$ is exactly $\mathfrak{p}$. The rest of the lemma follows trivially.

We return to the proof of Theorem 4.4. It is clear that the implication $(c) \Rightarrow(b)$ is a special case of the following.

Proposition 4.6. Let $R$ be an integrally closed local domain. If $E$ is a finitely generated $R$-module such that $\operatorname{Hom}(E, E)=\operatorname{Hom}(E, E)^{* *}$ and $\operatorname{Ext}_{R}^{1}(E, E)=0$, then $E=E^{* *}$.

Proof. Since $\operatorname{Hom}(E, E)=\operatorname{Hom}(E, E)^{* *}$, it follows that $\operatorname{Hom}(E, E)$ is torsion-free and therefore, by the previous lemma, that $E$ is torsion-free. Thus we have an exact sequence $0 \rightarrow E \rightarrow{ }^{*} E^{* *} \rightarrow B \rightarrow 0$, where $\psi: E \rightarrow E^{* *}$ is given by $\psi(e)(g)=g(e)$ for $e \in E$ and $g \in E^{*}$, and $B=E^{* *} / E$.

If $\operatorname{dim} R=0$, then $R$ is a field and the proposition is trivial. Suppose the proposition is true for all integrally closed local domains of dimension $<k$ (where $k \geqq 1$ ), and suppose $\operatorname{dim} R=k$. Since the hypothesis is preserved under localization with respect to any prime ideal in $R$, the induction hypothesis shows that $B \otimes R_{\mathfrak{p}}=0$ for every prime ideal $\mathfrak{p}$ of $R$ other than the maximal ideal $\mathfrak{m}$ of $R$. Therefore $B$ is annihilated by some power of $\mathfrak{m}$, and consequently $B$ has finite length. Thus, in order to show that $B$ is 0 , it suffices 
to show that $\operatorname{Hom}(E, B)=0$. For, if $B \neq 0$ and has finite length, then $\operatorname{Hom}(E / \mathfrak{m} E, B) \neq 0$ while at the same time $\operatorname{Hom}(E / \mathfrak{m} E, B)$ is contained in $\operatorname{Hom}(E, B)$. We shall now show that $\operatorname{Hom}(E, B)=0$.

From the exact sequence $0 \rightarrow E \rightarrow \psi E^{* *} \rightarrow B \rightarrow 0$ we deduce the exact sequence

$$
0 \rightarrow \operatorname{Hom}(E, E) \stackrel{\psi^{*}}{\rightarrow} \operatorname{Hom}\left(E, E^{* *}\right) \rightarrow \operatorname{Hom}(E, B) \rightarrow \operatorname{Ext}^{1}(E, E)
$$

Since $\operatorname{Ext}^{1}(E, E)=0$, it is sufficient to show that $\psi^{*}$ is an isomorphism, in order to conclude that $\operatorname{Hom}(E, B)=0$. But it is easy to verify that the map $\psi^{*}$ can be factored as follows:

$$
0 \rightarrow \operatorname{Hom}(E, E) \rightarrow \operatorname{Hom}(E, E)^{* *} \stackrel{\sigma^{*}}{\rightarrow}\left(E^{*} \otimes E\right)^{*} \rightarrow \operatorname{Hom}\left(E, E^{* *}\right),
$$

where $\operatorname{Hom}(E, E) \rightarrow \operatorname{Hom}(E, E)^{* *}$ is the usual imbedding of a torsion-free module into its second dual, and $\sigma^{*}$ is the map defined in Proposition 4.1. Since by hypothesis $\operatorname{Hom}(E, E)=\operatorname{Hom}(E, E)^{* *}$, while the other maps are isomorphisms by Proposition 4.1 , it follows that $\psi^{*}$ is an isomorphism. This completes the proof of Proposition 4.6, as well as the proof of the implication (c) $\Rightarrow$ (b) of Theorem 4.4.

We are now left with the implication $(b) \Rightarrow(a)$ of Theorem 4.4. Before presenting the details of its proof, we first review some definitions and results from the theory of local rings. For a fuller discussion, the reader is referred to [2]. If $R$ is a local ring and $E$ is a nonzero finitely generated $R$-module, then the codimension of $E$ (denoted by codim $E$ ) is defined to be the length of the longest sequence $x_{1}, x_{2}, \cdots, x_{2}$ of nonunits of $R$ such that $x_{i}$ is not a zero divisor in $E /\left(x_{1}, \cdots, x_{i-1}\right) E$. Then, codim $E$ is at most equal to $\operatorname{dim} R$ and is thus always finite. If $R$ is a regular local ring, then the relation codim $E$ thd $E=\operatorname{dim} R$ always holds. Thus a module $E$ over a regular local ring $R$ is free if, and only if, $\operatorname{codim} E=\operatorname{dim} R$.

Proposition 4.7. Let $R$ be a local ring, $A$ and $B$ finitely generated $R$-modules such that $\operatorname{Hom}(A, B) \neq 0$. If $\operatorname{codim} B \geqq i$, for $i=1,2$, then $\operatorname{codim} \operatorname{Hom}(A, B) \geqq i$.

Proof. Suppose that the nonunit $x$ is not a zero divisor in $B$. From the exact sequence $0 \rightarrow B \rightarrow \rightarrow^{x} B \rightarrow B / x B \rightarrow 0$ we deduce the exact sequence $0 \rightarrow \operatorname{Hom}(A, B) \rightarrow{ }^{x} \operatorname{Hom}(A, B) \rightarrow \operatorname{Hom}(A, B / x B)$, which shows that $x$ is not a zero divisor in $\operatorname{Hom}(A, B)$ and therefore that $\operatorname{codim} \operatorname{Hom}(A, B) \geqq 1$. If $y$ is not a zero divisor in $B / x B$, then $y$ is not a zero divisor in $\operatorname{Hom}(A, B / x B)$. $\operatorname{But} \operatorname{Hom}(A, B) / x \operatorname{Hom}(A, B)$ is a nonzero submodule of $\operatorname{Hom}(A, B / x B)$. Thus, if $\operatorname{codim} B \geqq 2$, then also $\operatorname{codim} \operatorname{Hom}(A, B) \geqq 2$, which proves the proposition.

COROllary. If $R$ is a regular local ring of dimension at most two, then 
$\operatorname{Hom}(E, R)$ is projective for every finitely generated $R$-module $E\left({ }^{8}\right)$. Thus, if $E=E^{* *}$, then $E$ is projective.

Proof. Since $\operatorname{codim} R=\operatorname{dim} R$ for a regular local ring $R$, the previous proposition shows that $\operatorname{codim} \operatorname{Hom}(E, R)=\operatorname{dim} R$ if $\operatorname{dim} R \leqq 2$ and $\operatorname{Hom}(E, R)$ $\neq 0$. Thus $\operatorname{Hom}(E, R)$ is always projective.

In view of this corollary, we see that Theorem 4.4 has been established for $\operatorname{dim} R \leqq 2$.

LEMmA 4.8. Let $R$ be a regular local ring of dimension at least three and let $B$ be a finitely generated $R$-module of codimension at least two. If $A$ is a finitely generated $R$-module such that $\operatorname{Hom}(A, B)$ is projective and $\operatorname{Ext}^{1}(A, B) \neq 0$, then codim $\operatorname{Ext}^{1}(A, B)>0$.

Proof. Let $0 \rightarrow \mathrm{B} \rightarrow^{x} B \rightarrow B / x B \rightarrow 0$ be exact with $x$ a nonunit in $R$. Then we have the exact sequence $0 \rightarrow \operatorname{Hom}(A, B) \rightarrow{ }^{x} \operatorname{Hom}(A, B) \rightarrow \operatorname{Hom}(A, B / x B)$ $\rightarrow \operatorname{Ext}^{1}(A, B) \rightarrow{ }^{x} \operatorname{Ext}^{1}(A, B)$. Suppose that codim $\operatorname{Ext}^{1}(A, B)=0$. Then $C=\operatorname{ker}\left(\operatorname{Ext}^{1}(A, B) \rightarrow{ }^{x} \operatorname{Ext}^{1}(A, B)\right)$ is not zero and also has codimension zero. Thus we have an exact sequence

$$
0 \rightarrow \operatorname{Hom}(A, B) / x \operatorname{Hom}(A, B) \rightarrow \operatorname{Hom}(A, B / x B) \rightarrow C \rightarrow 0 .
$$

Since $\operatorname{hd}_{R} C=\operatorname{dim} R \geqq 3$, and $\operatorname{hd}_{R}(\operatorname{Hom}(A, B) / x \operatorname{Hom}(A, B)) \leqq 1$, it follows that $\operatorname{hd}_{R} \operatorname{Hom}(A, B / x B)=\operatorname{dim} R$. But this means that $\operatorname{Hom}(A, B / x B) \neq 0$ and has codimension 0 . This is impossible, since codim $B \geqq 2$. (SeeProposition 4.7.)

As an immediate consequence of this lemma we have:

Proposition 4.9. If $R$ is a regular local ring, $E$ a finitely generated $R$ module such that $E=E^{* *}$ and $\operatorname{Hom}(E, E)$ is $R$-free, then $\operatorname{Ext}^{1}(E, E)=0$.

Proof. If $\operatorname{dim} R \leqq 2$, the proposition is trivially true since by the corollary to Proposition 4.7, $E$ is projective. Suppose that $\operatorname{dim} R=k>3$ and the proposition is true for rings of dimension less than $k$. Let $\mathfrak{p}$ be a prime ideal of $R$ other than the maximal ideal $\mathrm{m}$. Then $R_{\mathfrak{p}}$ is a regular local ring of dimension less than $k$, while $E \otimes R_{\mathfrak{p}}$ is equal to its second dual (with respect to $\left.R_{\mathfrak{p}}\right)$ and $\operatorname{Hom}_{R_{\mathfrak{p}}}\left(E \otimes R_{\mathfrak{p}}, E \otimes R_{\mathfrak{p}}\right)=R_{\mathfrak{p}} \otimes \operatorname{Hom}_{R}(E, E)$ is $R_{\mathfrak{p}}$-projective. Thus, $R_{\mathfrak{p}} \otimes \operatorname{Ext}^{1}(E, E)=\operatorname{Ext}_{R_{\mathfrak{p}}}^{1}\left(E \otimes R_{\mathfrak{p}}, E \otimes R_{\mathfrak{p}}\right)$ is 0 . Therefore $m^{\imath} \operatorname{Ext}^{1}(E, E)=0$ for some integer $t$, and consequently were $\operatorname{Ext}^{1}(E, E) \neq 0$, we would have codim $\operatorname{Ext}^{1}(E, E)=0$. In view of Lemma 4.8, we must have $\operatorname{Ext}^{1}(E, E)=0$.

Proposition 4.10. Let $R$ be a local ring, $E$ a finitely generated $R$-module of finite homological dimension $n$. If $A$ is any nonzero finitely generated $R$-module, then $\operatorname{Ext}_{R}^{n}(E, A) \neq 0$.

(3) It can be shown that this property actually characterizes local rings which are reqular and of dimension not greater than two. 
Proof. Let $0 \rightarrow X_{n} \rightarrow X_{n-1} \rightarrow \cdots \rightarrow X_{0} \rightarrow E \rightarrow 0$ be a minimal resolution of $E$ in which each $X_{i}$ is $R$-free and $\operatorname{Im}\left(X_{i+1} \rightarrow X_{i}\right) \subset \operatorname{mX} X_{i}$ for $i=0,1, \cdots, n-1$. Then we have the exact sequence

$$
\operatorname{Hom}\left(X_{n-1}, A\right) \rightarrow \operatorname{Hom}\left(X_{n}, A\right) \rightarrow \operatorname{Ext}^{n}(E, A) \rightarrow 0 .
$$

If $\operatorname{Ext}^{n}(E, A)=0$, then every $f$ in $\operatorname{Hom}\left(X_{n}, A\right)$ is the restriction to $X_{n}$ of some $g \in \operatorname{Hom}\left(X_{n-1}, A\right)$. Since $X_{n}$ is contained in $\mathfrak{m} X_{n-1}$, this would mean that $f\left(X_{n}\right) \subset \mathrm{m} A$ for every $f$ in $\operatorname{Hom}\left(X_{n}, A\right)$. Since $X_{n}$ is a nonzero free $R$-module, we find that $A=\mathrm{m} A$. This is impossible for a nonzero finitely generated $R$ module. Thus $\operatorname{Ext}^{n}(E, A) \neq 0$.

Suppose now that $R$ is a regular local ring of dimension three. If $E=E^{* *}$ and $\operatorname{Hom}_{R}(E, E)$ is $R$-free, we have several facts. Because codim $R=3$, it follows from Proposition 4.7 that codim $E \geqq 2$, or that hd $E \leqq 1$. At the same time, because of Proposition 4.9 we know that $\operatorname{Ext}^{1}(E, E)=0$. Therefore, because of Proposition 4.10 we cannot have hd $E=1$, so that hd $E$ must be 0 , and hence $E$ is free. This shows that the implication $(\mathrm{b}) \Rightarrow(\mathrm{a})$ of Theorem 4.4 is proved in case $\operatorname{dim} R \leqq 3$.

We now complete the proof of the implication (b) $\Rightarrow$ (a) of Theorem 4.4 by induction on $\operatorname{dim} R$. We suppose that $\operatorname{dim} R=k \geqq 4$, and that the result is valid for rings of dimension less than $k$. Assuming that $E=E^{* *}$ and that $\operatorname{Hom}_{R}(E, E)$ is $R$-free, we shall prove that $E$ is $R$-free.

Let $x$ be in $\mathfrak{m}$, not in $\mathfrak{m}^{2}$. Then $x$ is not a zero divisor in $E=E^{* *}$. From the exact sequence $0 \rightarrow E \rightarrow^{x} E \rightarrow E / x E \rightarrow 0$, we deduce the exact sequence $0 \rightarrow \operatorname{Hom}(E, E) \rightarrow{ }^{x} \operatorname{Hom}(E, E) \rightarrow \operatorname{Hom}(E, E / x E) \rightarrow \operatorname{Ext}^{1}(E, E)$. By Proposition 4.9, $\operatorname{Ext}^{1}(E, E)=0$, so that $\operatorname{Hom}_{R}(E, E / x E) \cong \operatorname{Hom}(E, E) / x \operatorname{Hom}(E, E)$. If we put $\bar{R}=R / x R$, we have therefore that $\operatorname{Hom}_{R}(E, E / x E)$ is a free $\bar{R}$-module. It is easily seen that $\operatorname{Hom}_{R}(E, E / x E)=\operatorname{Hom}_{\bar{B}}(\bar{E}, \bar{E})$, with $\bar{E}=E / x E$. Hence, by Lemma 4.5 we know that $\bar{E}$ is a torsion-free $\bar{R}$-module because $\operatorname{Hom}_{\bar{R}}(\bar{E}, \bar{E})$ is a torsion-free $\bar{R}$-module being $\bar{R}$-free. Denoting $\operatorname{Hom}_{\bar{R}}(\bar{E}, \bar{R})$ by $\bar{E}^{*}$, we know by Proposition 4.1 that $\operatorname{Hom}_{\bar{R}}(\bar{E}, \bar{E})^{* *} \cong \operatorname{Hom}_{\bar{R}}\left(\bar{E}^{*}, \bar{E}^{*}\right)$ since $\bar{R}$ is regular and therefore integrally closed. Thus $\operatorname{Hom}_{\bar{R}}\left(\bar{E}^{*}, \bar{E}^{*}\right)$ is $\bar{R}$-free and $\bar{E}^{*}=\left(\bar{E}^{*}\right)^{* *}$. Therefore, by the induction hypothesis, we have $\bar{E}^{*}$ is $\bar{R}$-free.

By a standard change of rings argument, we have $\operatorname{Ext}_{\bar{R}}(\bar{E}, \bar{R}) \cong \operatorname{Ext}_{R}^{1}(E, \bar{R})$ for $i \geqq 0$. Since $\operatorname{dim} R \geqq 4$, we have $\operatorname{dim} \bar{R} \geqq 3$, so that by Lemma 4.8 , $\operatorname{codim} \operatorname{Ext}_{\bar{k}}(\bar{E}, \bar{R})>0$ if $\operatorname{Ext}_{\bar{k}}(\bar{E}, \bar{R}) \neq 0$. Thus, if $\operatorname{Ext}_{R}^{1}(E, \bar{R}) \neq 0$, then codim $\operatorname{Ext}_{R}^{1}(E, \bar{R})>0$.

From the exact sequence $0 \rightarrow R \rightarrow^{x} R \rightarrow \bar{R} \rightarrow 0$ we deduce the exact sequence

$$
\begin{aligned}
0 \rightarrow E^{*} \stackrel{x}{\rightarrow} E^{*} \rightarrow \operatorname{Hom}_{R}(E, R) & \underset{x}{\rightarrow} \operatorname{Ext}^{1}(E, R) \\
& \operatorname{Ext}^{1}(E, R) \rightarrow \operatorname{Ext}^{1}(E, \bar{R}) .
\end{aligned}
$$

Applying the induction hypothesis, if $\mathfrak{p}$ is any prime ideal in $R$ other than $\mathfrak{m}$, then $R_{\mathfrak{p}} \otimes \operatorname{Ext}^{1}(E, R)=\operatorname{Ext}_{R_{\mathfrak{p}}}^{1}\left(E \otimes R_{\mathfrak{p}}, R_{\mathfrak{p}}\right)=0$. Therefore $\operatorname{Ext}^{1}(E, R)$ has finite 
length. Assume for the moment that $\operatorname{Ext}^{1}(E, R) \neq 0$. Then,

$$
\operatorname{Ext}^{1}(E, R) / x \operatorname{Ext}^{1}(E, R)
$$

has finite nonzero length and is contained in $\operatorname{Ext}^{1}(E, \bar{R})$, so that $\operatorname{codim} \operatorname{Ext}^{1}(E, \bar{R})=0$. This contradicts the conclusion arrived at above, so that we must have $\operatorname{Ext}^{1}(E, R)=0$. Consequently, the sequence $0 \rightarrow E^{*} \rightarrow{ }^{x} E^{*}$ $\rightarrow \operatorname{Hom}_{\bar{R}}(\bar{E}, \bar{R}) \rightarrow 0$ is exact. Since $\operatorname{Hom}_{\bar{R}}(\bar{E}, \bar{R})=E^{*} / x E^{*}$ is $\bar{R}$-free, we conclude that $E^{*}$ is $R$-free. Since $E=E^{* *}$, we find that $E$ is $R$-free and the proof of Theorem 4.4 is complete.

It should be observed that Theorem 4.4 gives another proof that a regular local ring is a unique factorization domain. For, if $\mathfrak{p}$ is a minimal prime ideal in $R$, then $\operatorname{Hom}(\mathfrak{p}, \mathfrak{p})=R$ since $R$ is integrally closed, while $\mathfrak{p}^{* *}=\left(\mathfrak{p}^{-1}\right)^{-1}=\mathfrak{p}$ because $\mathfrak{p}$ is minimal. Hence by Theorem $4.4 \mathfrak{p}$ is a free $R$-module and is therefore principal, which means that $R$ is a unique factorization domain.

It should also be observed that Theorems 4.3 and 4.4 show that if $R$ is a regular domain of Krull dimension at most two, then every maximal order in a full matrix algebra is a projective $R$-module. However, this statement is not true in higher dimension. For, suppose that $R$ has dimension greater than two; then there is a nonprojective finitely generated $R$-module $E$ such that $E=E^{* *}$. Then $\Lambda=\operatorname{Hom}_{R}(E, E)$ is a maximal order in a full matrix algebra, and is not projective because of Theorem 4.4. It is an open question whether every central simple algebra over $K$, the quotient field of a regular local ring of dimension at least three, contains a maximal order which is a projective $R$-module.

Appendix. For the convenience of the reader, we present in this appendix some facts about projective modules and their endomorphism rings which are used in the body of the paper. Since a number of these results have already appeared in the literature (see for example Morita [7] and Curtis [5]) we shall omit the details of some of the proofs.

If $\Gamma$ is a ring and $E$ is a left $\Gamma$-module, there are a number of additional rings and modules associated with this pair. For example, $\operatorname{Hom}_{\Gamma}(E, E)$ is the endomorphism ring of $E$. Denoting $\operatorname{Hom}_{\Gamma}(E, E)$ by $\Omega$, we will consider $E$ a left $\Omega$-module through the operation $\omega x=\omega(x)$, where $\omega \in \Omega, x \in E$. The group $\operatorname{Hom}_{\Gamma}(E, \Gamma)$ is both a right $\Gamma$ and right $\Omega$-module by means of the following operations. If $f \in \operatorname{Hom}_{\Gamma}(E, \Gamma), \gamma \in \Gamma, x \in E$, then we define $\{f \gamma\}(x)=f(x) \gamma$; and for $\omega \in \Omega,\{f \omega\}(x)=f(\omega x)$. The operations of $\Gamma$ and $\Omega$ commute on $\operatorname{Hom}_{\Gamma}(E, \Gamma)$.

We define the map $\mu: \operatorname{Hom}_{\Gamma}(E, \Gamma) \otimes_{\Gamma} E \rightarrow \Omega$ as follows: $\mu(f \otimes x)(y)=f(y) x$. Because the operations of $\Omega$ and $\Gamma$ commute both on $E$ and on $\operatorname{Hom}_{\Gamma}(E, \Gamma)$, the tensor product $\operatorname{Hom}_{\Gamma}(E, \Gamma) \otimes_{\Gamma} E$ is a two-sided $\Omega$-module. It is clear that $\mu$ is a two-sided $\Omega$-mapping.

Proposition A.1. A necessary and sufficient condition that $E$ be a finitely 
generated projective $\Gamma$-module is that the map $\mu: \operatorname{Hom}_{\Gamma}(E, \Gamma) \otimes_{\Gamma} E \rightarrow \Omega$ be an epimorphism. If $\mu$ is an epimorphism, then $\mu$ is an isomorphism.

Proof. The proposition is a reformulation of the statement that $E$ is a finitely generated projective $\Gamma$-module, if and only if, there are elements $x_{1}, \cdots, x_{n} \in E$ and $f_{1}, \cdots, f_{n} \in \operatorname{Hom}_{\Gamma}(E, \Gamma)$ such that $\sum f_{i}(y) x_{i}=y$, all $y \in E$. (See [4, p. 132].)

The pairing of $\operatorname{Hom}_{\Gamma}(E, \Gamma)$ with $E$ to $\Gamma$ defines a map $\tau: \operatorname{Hom}_{\Gamma}(E, \Gamma) \otimes_{\Omega} E$ $\rightarrow \Gamma$ by $\tau(f \otimes x)=f(x)$, which is easily seen to be a two-sided $\Gamma$-map. The image of $\tau$ is thus a two-sided ideal in $\Gamma$ which will be called the trace ideal of $E$ in $\Gamma$ and will be denoted by $\mathfrak{I}_{\Gamma}(E)$. It will shortly be shown that $\mathfrak{T}_{\Gamma}(E)$ is connected with the properties of $E$ as an $\Omega$-module.

The fact that $\Gamma$ and $\Omega$ commute on $E$ gives rise to a ring homomorphism $i: \Gamma \rightarrow \operatorname{Hom}_{\Omega}(E, E)$, whose kernel $\mathfrak{a}(E)$ is the annihilator of $E$ in $\Gamma$. Finally we have a map $\psi: \operatorname{Hom}_{\Gamma}(E, \Gamma) \rightarrow \operatorname{Hom}_{\Omega}(E, \Omega)$ as follows: $\psi(f)(x)=\mu(f \otimes x)$.

After these preparatory remarks, we have:

THEOREM A.2.

(a) The diagram:

$$
\begin{array}{ccc}
\operatorname{Hom}_{\Gamma}(E, \Gamma) \otimes_{\Omega} E \stackrel{\psi \otimes 1}{\tau} \operatorname{Hom}_{\Omega}(E, \Omega) \otimes_{\Omega} E \\
\Gamma & \stackrel{i}{\longrightarrow} & \operatorname{Hom}_{\Omega}(E, E)
\end{array}
$$

is commutative.

(b) $\mathfrak{a}(E) \mathfrak{T}_{\Gamma}(E)=0$.

If $\mathfrak{a}(E)+\mathfrak{T}_{\mathrm{r}}(E)=\Gamma$, then:

(c) $\mu^{\prime}$ is an epimorphism, so that $E$ is a finitely generated projective $\Omega$ module.

(d) $i$ is an epimorphism and splits as a left $\Gamma$-map so that $\mathfrak{a}(E)$ is a left direct summand of $\Gamma$.

If $\mathfrak{T}_{\Gamma}(E)=\Gamma$, then:

(e) $\mathfrak{a}(E)=0$.

(f) $\psi$ is a monomorphism.

(g) All the maps in the diagram are isomorphisms.

(h) $\operatorname{Hom}_{\Gamma}(E, \Gamma)$ is a finitely generated projective $\Omega$-module.

Proof. (a) and (b). The statements follow directly from the definitions of the various objects.

(c) and (d). Since $E$ is a left $\operatorname{Hom}_{\Omega}(E, E)$-module with operations of $\operatorname{Hom}_{\Omega}(E, E)$ and $\Omega$ commuting on $E$, it follows that $\operatorname{Hom}_{\Gamma}(E, \Gamma) \otimes_{\Omega} E$ is also a left $\operatorname{Hom}_{\Omega}(E, E)$-module. The operations of $\Gamma$ on $\operatorname{Hom}_{\Gamma}(E, \Gamma) \otimes_{\Omega} E$ given by the mapping $i$ is the same as that given by $\Gamma$ on $E$. The hypothesis $\mathfrak{a}(E)+\mathfrak{T}_{\Gamma}(E)=\Gamma$ implies that there is an element $z \in \operatorname{Hom}_{\Gamma}(E, \Gamma) \otimes_{\Omega} E$ with 
$i \tau(z)=1$. Define $\alpha: \operatorname{Hom}_{\Omega}(E, E) \rightarrow \operatorname{Hom}_{\Gamma}(E, \Gamma) \otimes_{\Omega} E$ by $\alpha(x)=x z$. It is easily checked that $\alpha$ is a left $\Gamma$-map. Since $i \tau \alpha=$ identity, we have that $i$ is an epimorphism and splits as a left $\Gamma$-map. We also have that $\mu^{\prime}(\psi \otimes 1) \alpha=$ identity, so that $\mu^{\prime}$ is an epimorphism and consequently $E$ is a finitely generated projective $\Omega$-module.

(e) and (f). If $\mathfrak{I}_{\Gamma}(E)=\Gamma$, then (b) shows that $\mathfrak{a}(E)=0$. From this it follows readily that $\psi$ is a monomorphism.

(g). Because $E$ is a projective $\Omega$-module, the fact that $\psi$ is a monomorphism assures that $\psi \otimes 1$ is also a monomorphism. Combining this with the fact that $i$ and $\mu^{\prime}$ are isomorphisms and that $\tau$ is an epimorphism, it follows that the maps $i, \tau, \mu^{\prime}, \psi \otimes 1$ are isomorphisms.

(h) We shall apply Proposition A.1 to $\operatorname{Hom}_{\Gamma}(E, \Gamma)$ as a (right) $\Omega$-module. To do so, we first define a map $\beta: E \rightarrow \operatorname{Hom}_{\Omega}\left(\operatorname{Hom}_{\Gamma}(E, \Gamma), \Omega\right)$ by means of : $\beta(x)(f)=\mu(f \otimes x)$. This leads to the diagram:

$$
\begin{array}{ccc}
\operatorname{Hom}_{\Gamma}(E, \Gamma) \otimes_{\Omega} E & \stackrel{1 \otimes \beta}{\longrightarrow} \operatorname{Hom}_{\Gamma}(E, \Gamma) \otimes_{\Omega} \operatorname{Hom}_{\Omega}\left(\operatorname{Hom}_{\Gamma}(E, \Gamma), \Omega\right) \\
\tau \downarrow & \stackrel{i^{\prime}}{\longrightarrow} & \operatorname{\mu om}_{\Omega}^{\prime \prime}\left(\operatorname{Hom}_{\Gamma}(E, \Gamma), \operatorname{Hom}_{\Gamma}(E, \Gamma)\right)
\end{array}
$$

in which the map $i^{\prime}$ is defined analogously to $i$. One checks readily that the diagram commutes. Since $\tau$ is an epimorphism, the image of $i^{\prime} \tau$ contains 1 , so that 1 is in the image of $\mu^{\prime \prime}$. It follows by Proposition A.1 that $\operatorname{Hom}_{\Gamma}(E, \Gamma)$ is a finitely generated projective $\Omega$-module.

Proposition A.3. If $E$ is a finitely generated projective $\Gamma$-module and $\Omega=\operatorname{Hom}_{\Gamma}(E, E)$, then $\mathfrak{T}_{\Gamma}(E) E=E$ and $\mathfrak{T}_{\Omega}(E)=\Omega$. If in addition $\Gamma$ is commutative, then $\mathfrak{a}(E)+\mathfrak{T}_{\Gamma}(E)=\Gamma$, and consequently, $E$ is a finitely generated projective $\Omega$-module.

Proof. The statements follow from the definition of the trace ideal combined with the remark immediately following the statement of Proposition A.1.

Remark. The part of Proposition A.3 concerned with the case in which $\Gamma$ is commutative is not in general true without that assumption. That is, there exist finitely generated faithful projective $\Gamma$-modules $E$ with $\mathfrak{T}_{\Gamma}(E) \neq \Gamma$ and $E$ not $\Omega$-projective.

If $E$ is a left $\Gamma$-module and $\Omega=\operatorname{Hom}_{\Gamma}(E, E)$, we denote by $\mathfrak{N (}(\Gamma)$ the category of right $\Gamma$-modules and by $\mathfrak{T}(\Omega)$ the category of left $\Omega$-modules. If $M \in \mathscr{T}(\Omega)$ define $\mathcal{F}(M)=\operatorname{Hom}_{\Gamma}(E, \Gamma) \otimes_{\Omega} M$. Then, $\mathcal{F}(M)$ is a right $\Gamma$-module because $\operatorname{Hom}_{\Gamma}(E, \Gamma)$ is a right $\Gamma$-module and the operations of $\Gamma$ and $\Omega$ commute on $\operatorname{Hom}_{\Gamma}(E, \Gamma)$. Thus, $\mathcal{F}(M) \in \mathscr{T}(\Gamma)$. If $M, M^{\prime} \in \mathscr{T}(\Omega)$ and $\phi: M \rightarrow M^{\prime}$ is an $\Omega$-mapping, we define $\mathcal{F}(\phi): \mathcal{F}(M) \rightarrow \mathcal{F}\left(M^{\prime}\right)$ by $\mathcal{F}(\phi)=1 \otimes \phi$. These definitions serve to show that $\mathcal{F}$ is a covariant additive functor from $\mathfrak{T}(\Omega)$ to $\mathscr{T}(\Gamma)$. Similarly, if $N \in \mathscr{T}(\Gamma)$, we set $\mathcal{G}(N)=N \otimes_{\Gamma} E$. Here $\mathcal{G}(N)$ is a 
left $\Omega$-module because $E$ is a left $\Omega$-module, and the operations of $\Omega$ and $\Gamma$ commute on $E$. If $\phi: N \rightarrow N^{\prime}$ is a $\Gamma$-map, then $\mathcal{G}(\phi): \mathcal{G}(N) \rightarrow \mathcal{G}\left(N^{\prime}\right)$ is defined to be $\phi \otimes 1$. With these definitions, $\mathcal{G}$ is a covariant additive functor from $\mathfrak{T}(\Gamma)$ to $\operatorname{Tr}(\Omega)$.

PROPOSITION A.4. F and $\mathcal{G}$ are covariant additive functors; for $M \in \mathscr{T}(\Omega)$, we have that $\mathcal{G F}(M)=\left(\operatorname{Hom}_{\Gamma}(E, \Gamma) \otimes_{\Gamma} E\right) \otimes_{\Omega} M$, while for $N \in \mathfrak{N}(\Gamma)$, we have $F \mathcal{G}(N)=N \otimes_{\Gamma}\left(\operatorname{Hom}_{\Gamma}(E, \Gamma) \otimes_{\Omega} E\right)$. If $E$ is a finitely generated projective $\Gamma$ module, then $\mathcal{G}$ is exact and $\mathcal{G F}$ is the identity. If $\mathfrak{T}_{\Gamma}(E)=\Gamma$, then $\mathcal{F}$ is exact and FG is the identity.

Proof. The statements result from combining Proposition A.1 and Theorem A. 2 with the definitions of $\mathcal{F}$ and $\mathcal{G}$.

There are several special cases of Proposition A.4 of sufficient importance to be stated separately:

TheOREM A.5. If $\mathfrak{T}_{\Gamma}(E)=\Gamma$ then $N \otimes_{\Gamma} E=0$ for a right $\Gamma$-module $N$ implies $N=0$. If $E$ is a finitely generated projective $\Gamma$-module and $\mathfrak{T}_{\Gamma}(E)=\Gamma$ then $\mathcal{F}$ and $\mathcal{G}$ establish a one-to-one correspondence between the two-sided ideals in $\Omega$ and the two-sided ideals in $\Gamma$ which preserves inclusion and preserves projectivity (left $\Omega$ and right $\Gamma$ ).

Proof. The proof follows directly from Proposition A.4. It might be of interest to have an explicit description of the correspondence between the ideals of $\Omega$ and of $\Gamma$. If $\mathfrak{l}$ is a two-sided ideal of $\Omega$, we form $\mathcal{F}\left(\mathfrak{l} \otimes_{\Omega} E\right)$ $=\operatorname{Hom}_{\Gamma}(E, \Gamma) \otimes_{\Omega} \mathfrak{l} \otimes_{\Omega} E$. The monomorphism $0 \rightarrow \mathfrak{I} \rightarrow \Omega$ induces a monomorphism $\operatorname{Hom}_{\Gamma}(E, \Gamma) \otimes_{\Omega} \mathfrak{l} \otimes_{\Omega} E \rightarrow \operatorname{Hom}_{\Gamma}(E, \Gamma) \otimes_{\Omega} E=\Gamma$, so that $\operatorname{Hom}_{\Gamma}(E$, $\Gamma) \otimes_{\Omega} l \otimes_{\Omega} E$ is naturally isomorphic to a two-sided ideal of $\Gamma$.

Suppose now that $E$ is a finitely generated projective $\Gamma$-module and $\mathfrak{T}_{\Gamma}(E)=\Gamma$. If $\Omega=\operatorname{Hom}_{\Gamma}(E, E)$, then we know by Theorem A.2 and Proposition A.3 that $E$ is symmetric in relation to $\Gamma$ and $\Omega$, that is, $\Gamma=\operatorname{Hom}_{\Omega}(E, E)$, and $E$ is a finitely generated projective $\Omega$-module with $\mathfrak{T}_{\Omega}(E)=\Omega$. Also, Theorem A.2 shows that the map $\psi: \operatorname{Hom}_{\Gamma}(E, \Gamma) \rightarrow \operatorname{Hom}_{\Omega}(E, \Omega)$ is a monomorphism. Using the symmetry, we have also a map $\psi^{\prime}: \operatorname{Hom}_{\Omega}(E, \Omega)$ $\rightarrow \operatorname{Hom}_{\Gamma}(E, \Gamma)$ and there is no difficulty in verifying that $\psi$ and $\psi^{\prime}$ are inverses of each other.

Proposition A.6. If $E$ is a finitely generated projective $\Gamma$-module with $\mathfrak{T}_{\Gamma}(E)=\Gamma$ then $\psi: \operatorname{Hom}_{\Gamma}(E, \Gamma) \rightarrow \operatorname{Hom}_{\Omega}(E, \Omega)$ is an isomorphism. If $M$ is $a$ left $\Omega$-module, then the mapping $\rho: \operatorname{Hom}_{\Omega}(E, M) \otimes_{\Gamma} E \rightarrow M$ defined by $\rho(f)(x)$ $=f(x)$ is an isomorphism.

Proof. The first part of the statement follows from the remarks immediately above. For the mapping $\rho$ we have the following situation. By Proposition A.4, we have $M=\mathcal{G F}(M)=\mathcal{F}(M) \otimes_{\Gamma} E$ and $\mathcal{F}(M)=\operatorname{Hom}_{\Gamma}(E, \Gamma) \otimes_{\Omega} M$ $\cong \operatorname{Hom}_{\Omega}(E, \Omega) \otimes_{\Omega} M$. The latter isomorphism is $\psi \otimes 1$. Now it is easily checked 
that $\operatorname{Hom}_{\Omega}(E, \Omega) \otimes_{\Omega} M \cong M$ when $E$ is a finitely generated projective $\Omega$ module. Composing these various isomorphisms shows that $\rho$ is also an isomorphism.

\section{REFERENCES}

1. M. Auslander, On the dimensions of modules and algebras (III), Nagoya Math. J. vol. 9 (1955) pp. 67-77.

2. M. Auslander and D. A. Buchsbaum, Homological dimension in local rings, Trans. Amer. Math. Soc. vol. 85 (1957) pp. 390-405.

3. —_, Ramification theory in noetherian rings, Amer. J. Math. vol. 81 (1959) pp. 749764.

4. H. Cartan and S. Eilenberg, Homological algebra, Princeton, 1956.

5. C. W. Curtis, On commuting rings of endomorphisms, Canad. J. Math. vol. 8 (1956) pp. 271-291.

6. M. Deuring, Algebren, Berlin, Springer, 1935.

7. K. Morita, Duality for modules, Science reports of the Tokyo Kyoiku Daigaku, Sec. A, vol. 6 no. 150 (1958).

BRANDEIS UNIVERSITY,

WalthaM, MassachusetTs 